\title{
The limits on the strong Higgs sector parameters in the presence of new vector resonances
}

\author{
Mikuláš Gintner ${ }^{1,2, a} \mathbb{D}$, Josef Juráńn ${ }^{2,3, b}$ \\ ${ }^{1}$ Physics Department, University of Zilina, Univerzitná 1, 01026 Zilina, Slovakia \\ ${ }^{2}$ Institute of Experimental and Applied Physics, Czech Technical University in Prague, Horská 3a/22, 12800 Prague, Czech Republic \\ ${ }^{3}$ Institute of Physics, Silesian University in Opava, Bezručovo nám. 13, 74601 Opava, Czech Republic
}

Received: 2 August 2016 / Accepted: 6 November 2016 / Published online: 28 November 2016

(C) The Author(s) 2016. This article is published with open access at Springerlink.com

\begin{abstract}
In this paper, we investigate how the LHC data limit the Higgs-related couplings in the effective description of a strongly interacting extension of the Standard model. The Higgs boson is introduced as a scalar composite state and it is followed in the mass hierarchy by an $S U$ (2) triplet of vector composites. The limits are calculated from the constraints obtained in the recent ATLAS+CMS combined analysis of the data from 2011 and 2012. We find that the data prefer the scenario where the Higgs couplings to the electroweak gauge bosons differ from its couplings to the vector triplet. We also investigate the unitarity limits of the studied effective model for the experimentally preferred values of the Higgs couplings. We find from the $\pi \pi \rightarrow \pi \pi$ scattering amplitudes that for the vector resonance masses between one and two $\mathrm{TeV}$ significant portions of the experimentally allowed regions are well below the unitarity limit. We also evaluate how the existing ATLAS and CMS Run-2 data restrict our model with the upper bounds on the resonance production cross section times its branching ratio for various decay channels. The masses in the range $1 \mathrm{TeV} \leq M_{\rho} \leq 2 \mathrm{TeV}$ are not excluded in parts or even full parameter space of our theory.
\end{abstract}

\section{Introduction}

Even though the LHC experiments ATLAS and CMS achieved a spectacular success by discovering the $125 \mathrm{GeV}$ Higgs boson $[1,2]$ it was more the beginning rather than the end of the struggle to uncover the character of physics beyond the Standard model (SM). To this moment, it has not even been settled down whether new physics takes the form of

\footnotetext{
a e-mail: gintner@fyzika.uniza.sk

b e-mail: josef.juran@utef.cvut.cz
}

weakly coupled supersymmetry or strongly coupled composites.

In the strongly coupled scenario, the observed lightness of the Higgs boson with respect to the expected size of the compositeness scale (naively, $\Lambda=4 \pi v \simeq 3 \mathrm{TeV}$ ) could be explained if the Higgs boson were a (pseudo)NambuGoldstone boson after a breakdown of suitable extended global symmetry [3-10]. Another widely studied possibility is to embed the composite Higgs in an effective $S U(2)$ doublet [11-27] where its lightness would be guaranteed by the theory's particularities.

If the Higgs is generated as a composite state by new strong interactions the extension of the SM can be effectively described by higher dimensional operators that do not decouple in the low-energy limit. Presumably, they would modify the SM couplings of the Higgs boson with the heavy SM fields, such as the electroweak (EW) gauge bosons and/or the third quark generation. However, while the light SM Higgs boson can guarantee unitarity of the SM to virtually arbitrary high energies, this is not true anymore if the Higgs couplings become anomalous [28-30]. Nevertheless, the least one could require from the successful effective description of the composite state phenomenology is that it will not break down at energy below the compositeness scale. Meeting this expectation might be assisted with by the presence of additional new composite states which naturally occur in strongly interacting theories, anyway. Any further progress in dealing with these questions largely depends on an experimental input. Therefore, it is interesting to find what the most recent measurements of the Higgs boson couplings imply for various effective descriptions of strongly interacting extensions of the SM.

In this paper, we calculate the LHC limits on the effective theory describing possible early signs of strongly interacting physics beyond the SM. The effective description we work with is a rather simplistic view of what might be observed at 
the LHC beyond the $125 \mathrm{GeV}$ Higgs boson. We work with the vision where the Higgs boson is a scalar composite state followed in the mass hierarchy by a vector composite $S U(2)$ triplet state.Our approach is closely related to the formalism used in [31].

In particular, the Higgs sector of the effective Lagrangian under consideration is based on the non-linear sigma model with the $125-\mathrm{GeV} S U(2)_{L+R}$ scalar singlet complementing the non-linear triplet of the Nambu-Goldstone bosons. The new vector resonances are explicitly present in the form of an $S U(2)_{L+R}$ triplet. The vector triplet is introduced as a gauge field via the hidden local symmetry approach [32]. Consequently, it mixes with the EW gauge bosons. It results in the appearance of the mixing-generated (indirect) couplings of the vector triplet with all SM fermions. This setup fits the situation when the global $S U(2)_{L} \times S U(2)_{R}$ symmetry is broken down to $S U(2)_{L+R}$. The gauge sector of this effective description is equivalent to the gauge sector of highly deconstructed Higgsless model with only three sites [33].

The above-mentioned effective scenario has also been a basis for the effective Lagrangian we introduced and investigated in $[34,35] .{ }^{1}$ Therein, the vector triplet couples directly to the third quark doublet only and to none of the other SM fermions. In addition, even the interactions to the right quarks are not necessarily universal. Similar interaction patterns can be found in various recent extensions of the SM, including the partial compositeness and extra-dimensional scenarios. As was shown in $[34,35]$ this arrangement helps relax the tight restrictions placed by the electroweak precision data on the vector triplet coupling to the light SM fermions, the bottom quark included. While the study in this paper follows our tBESS model (and, in the relevant parts, matches the model considered in [31]), its conclusions will also be partly, or fully, applicable to a wider class of effective models, e.g., with different fermionic sectors.

The question we address in this paper concerns a possible structure of the interactions between the new vector triplet and the Higgs boson. Under the considered symmetry, the Higgs boson coupling to the new vector triplet can differ from the Higgs couplings to the EW gauge bosons. This splitting might appear as an unwelcome complication. Nevertheless, as we will demonstrate in the paper, the ATLAS and CMS data support it. In particular, the $H \rightarrow \gamma \gamma$ constraint is the key component of the data that makes it difficult for the nosplitting scenario to satisfy the LHC measurements.

Using the results of $[31,34,35]$ we also analyze the treelevel unitarity limits of our model resulting from the scattering of the longitudinal EW gauge bosons for the allowed values of the Higgs couplings obtained in this work. When

\footnotetext{
1 We call it the tBESS model to stress its connection to the BESS model [36-38]. The name modifying "t" suggests a special standing of the top-quark related doublet in the model.
}

authors of [31] addressed unitarity questions the discovery of the Higgs boson was not confirmed yet. Thus, while they used the correct mass of the Higgs boson in their analysis, they were lacking any of the experimental input on the Higgs interactions available to us today. This was compensated for by the usage of the sum rules. However, one of the used sum rules imposes the no-spitting condition. The results of our analysis compel us to abandon this assumption and calculate the unitarity limits under different conditions.

This paper is organized as follows. In Sect. 2, we introduce the necessary components of the effective Lagrangian and work out the consequences of the Higgs-to-gauge-boson coupling splitting. Section 3 is devoted to the calculations of the constraints for the Higgs-to-gauge-boson couplings. Particularly, in Sect. 3.1, we set up the framework for the constraint calculations. In Sect. 3.2, we demonstrate the tension between the $H \rightarrow \gamma \gamma$ and other LHC measurement constraints for the model parameters when the universality of the Higgs-to-gauge-boson couplings is assumed. In Sect. 3.3, we calculate the best fits and constraints for the Higgs-to-gauge-boson parameters when the Higgs coupling to the new vector triplet can differ from the Higgs coupling to the EW gauge bosons. In Sect. 3.4 we investigate the unitarity limits for our model. Finally, in Sect. 4, we investigate the applicability of the existing LHC limits on the masses of new vector resonances to our model. We compare the predictions of our model for the production cross section of the vector resonance times its branching ratio for various decay channels with the existing experimental upper bounds obtained by the ATLAS and CMS Collaborations. Section 5 presents the conclusions of the paper.

\section{The effective Lagrangian}

The effective Lagrangian is built to respect the global $S U(2)_{L} \times S U(2)_{R} \times U(1)_{B-L} \times S U(2)_{H L S}$ symmetry of which the $S U(2)_{L} \times U(1)_{Y} \times S U(2)_{H L S}$ subgroup is also a local symmetry. The $S U(2)_{H L S}$ symmetry is an auxiliary gauge symmetry invoked to accommodate the $S U(2)$ triplet of vector resonances. Beside the scalar singlet $h(x)$ and the vector triplet $\mathbf{V}_{\mu}=\left(V_{\mu}^{1}, V_{\mu}^{2}, V_{\mu}^{3}\right)$, the effective Lagrangian is built out of the SM fields only.

The Lagrangian can be split in three terms ${ }^{2}$

$\mathcal{L}=\mathcal{L}_{\mathrm{GB}}+\mathcal{L}_{\mathrm{ESB}}+\mathcal{L}_{\text {ferm }}$,

where $\mathcal{L}_{\mathrm{GB}}$ describes the gauge-boson sector including the $S U(2)_{\mathrm{HLS}}$ triplet,

\footnotetext{
2 While the full formulation of the model can be found in [35], the definitions of basic quantities used in Eqs. (2) through (5) are, for the reader's convenience, summarized in Appendix.
} 


$$
\begin{aligned}
\mathcal{L}_{\mathrm{GB}}= & \frac{1}{2 g^{2}} \operatorname{Tr}\left(\boldsymbol{W}_{\mu \nu} \boldsymbol{W}^{\mu \nu}\right)+\frac{1}{2 g^{\prime 2}} \operatorname{Tr}\left(\boldsymbol{B}_{\mu \nu} \boldsymbol{B}^{\mu \nu}\right) \\
& +\frac{2}{g^{\prime \prime 2}} \operatorname{Tr}\left(\boldsymbol{V}_{\mu \nu} \boldsymbol{V}^{\mu \nu}\right),
\end{aligned}
$$

$\mathcal{L}_{\mathrm{ESB}}$ is the scalar sector responsible for spontaneous breaking of the electroweak and hidden local symmetries, and $\mathcal{L}_{\text {ferm }}$ is the fermion Lagrangian of the model.

Let us express $\mathcal{L}_{\text {ESB }}$ as a sum of two terms, $\mathcal{L}_{\text {ESB }}=\mathcal{L}_{h}+$ $\mathcal{L}_{\mathrm{hV}}$, where

$\mathcal{L}_{h}=\frac{1}{2} \partial_{\mu} h \partial^{\mu} h-\frac{1}{2} M_{h}^{2} h^{2}-c_{h} \frac{M_{h}^{2}}{2 v} h^{3}-c_{h}^{\prime} \frac{M_{h}^{2}}{8 v^{2}} h^{4}$

contains the kinetic term and the self-interactions of the Higgs boson with the mass $M_{h}=125 \mathrm{GeV}$ and free parameters $c_{h}$ and $c_{h}^{\prime}$. Furhter,

$$
\begin{aligned}
\mathcal{L}_{\mathrm{hV}}= & -v^{2}\left[\operatorname{Tr}\left(\bar{\omega}^{\perp}\right)^{2}\left(1+2 a_{V} \frac{h}{v}+a_{V}^{\prime} \frac{h^{2}}{v^{2}}+\ldots\right)\right. \\
& \left.-v^{2}+\alpha \operatorname{Tr}\left(\bar{\omega}^{\|}\right)^{2}\left(1+2 a_{\rho} \frac{h}{v}+a_{\rho}^{\prime} \frac{h^{2}}{v^{2}}+\ldots\right)\right]
\end{aligned}
$$

is responsible for the masses of all gauge bosons including the new vector triplet, and describes their interactions with the Higgs boson. The interactions are parameterized by the free parameters $a_{V}, a_{\rho}, a_{V}^{\prime}, a_{\rho}^{\prime}, \ldots$. Below, only the interaction terms of (4) that are at most linear in $h$ will be considered. ${ }^{3}$ Terms with higher powers of $h$, which are not important for higgs phenomenology at the LHC, can be restored at any time without affecting our conclusions.

The fermion sector $\mathcal{L}_{\text {ferm }}$ in its minimal formulation contains the fermionic kinetic terms and the fermion interactions with the EW gauge bosons as well as terms responsible for the couplings of the SM fermions to the Higgs boson. While the EW part is kept identical to the SM one, the interactions of the Higgs boson with the fermions can assume non-SM values. Their parameterization is based on the following interaction Lagrangian:

$$
\mathcal{L}_{\text {ferm }}^{\text {scalar }}=-\sum_{k=1}^{6} \bar{\psi}_{L}^{k} U M_{f}^{k}\left(1+c_{f}^{k} \frac{h}{v}+c_{f}^{\prime k} \frac{h^{2}}{v^{2}}+\ldots\right) \psi_{R}^{k}+\text { h.c. }
$$

where $M_{f}^{k}$ is a $2 \times 2$ diagonal matrix with the masses of the upper and bottom $k^{\text {th }}$ fermion doublet components on its diagonal, and $U=\xi(\boldsymbol{\pi}) \cdot \xi(\pi)=\exp (2 i \pi \boldsymbol{\tau} / v)$. Note that when $c_{k}=1, \forall k$, and the rest of $c$ 's are zeros the scalar resonance interactions with fermions imitate the corresponding

\footnotetext{
${ }^{3}$ Although the quadratic terms would be needed if one wished to maintain the possibility to eliminate the linear growth in $s$ from the scattering amplitude $\pi \pi \rightarrow h h$ and thus improve the unitarity limit for the model.
}

interactions of the SM Higgs boson. Again, only the interaction terms that are at most linear in $h$ will be considered below.

Possible direct interactions of the fermions with the vector triplet do not play a role in the calculation of the limits investigated in this paper. Thus, this part of the effective Lagrangian is left unspecified. Nevertheless, we would like to mention the example of the setup of this sector where only the third quark generation couples directly to the vector triplet and the interaction of the right top quark is disentangled from the interaction of the right bottom quark. We suggested and analyzed the effective model with this kind of the fermion sector in $[34,35]$. For the sake of completeness, we would like to point out that in this model the vector resonances do interact with the SM fermions, including the light ones, even if there are no direct interactions introduced. This is due to the mixing between the EW gauge bosons and the vector resonance triplet. Of course, the mixing-induced couplings are suppressed by the transformation matrix elements; they are proportional to $1 / g^{\prime \prime}$.

The mixing of the gauge fields occurs in the process of diagonalization of the gauge-boson mass matrix. After gauging out all six Goldstone bosons the Lagrangian $\mathcal{L}_{\mathrm{hV}}$ reads

$\mathcal{L}_{\mathrm{hV}}=\mathcal{M}(\alpha)+\frac{2 a_{V}}{v} \mathcal{M}(\alpha r) h$,

where $r=a_{\rho} / a_{V}$ and where

$$
\begin{aligned}
\mathcal{M}(\alpha) \equiv & \frac{1}{2}\left(X_{\mu}^{-}\right)^{\dagger} \cdot \boldsymbol{M}_{\mathbf{C}}^{\mathbf{2}}(\alpha) \cdot X_{\mu}^{-}+\frac{1}{2}\left(X_{\mu}^{+}\right)^{\dagger} \cdot \boldsymbol{M}_{\mathbf{C}}^{\mathbf{2}}(\alpha) \cdot X_{\mu}^{+} \\
& +\frac{1}{2}\left(X_{\mu}^{0}\right)^{\dagger} \cdot \boldsymbol{M}_{\mathbf{N}}^{\mathbf{2}}(\alpha) \cdot X_{\mu}^{0}
\end{aligned}
$$

is the gauge-boson mass term. Further, $X^{ \pm}=\left(W_{f}^{ \pm}, V^{ \pm}\right)^{T}$, $X^{0}=\left(W^{3}, B, V^{3}\right)^{T}$, and $\boldsymbol{M}_{\mathbf{C}}^{\mathbf{2}}(\alpha)$ and $\boldsymbol{M}_{\mathbf{N}}^{\mathbf{2}}(\alpha)$ are the squared-mass matrices of the charged and neutral gauge bosons, respectively,

$$
\begin{aligned}
& \boldsymbol{M}_{\mathbf{C}}^{2}=\frac{v^{2}}{4}\left(\begin{array}{cc}
(1+\alpha) g^{2} & -\alpha g g^{\prime \prime} \\
-\alpha g g^{\prime \prime} & \alpha g^{\prime \prime 2}
\end{array}\right), \\
& \boldsymbol{M}_{\mathbf{N}}^{2}=\frac{v^{2}}{4}\left(\begin{array}{ccc}
(1+\alpha) g^{2} & -(1-\alpha) g g^{\prime} & -\alpha g g^{\prime \prime} \\
-(1-\alpha) g g^{\prime} & (1+\alpha) g^{\prime 2} & -\alpha g^{\prime} g^{\prime \prime} \\
-\alpha g g^{\prime \prime} & -\alpha g^{\prime} g^{\prime \prime} & \alpha g^{\prime \prime 2}
\end{array}\right) .
\end{aligned}
$$

The diagonalization process results in the transformation of the gauge-boson basis, from the mass one to the flavor one, $\{Y\} \rightarrow\{X\}:$

$X_{\mu}^{ \pm}=O^{(C)} \cdot Y_{\mu}^{ \pm}, \quad X_{\mu}^{0}=O^{(N)} \cdot Y_{\mu}^{0}$,

where $Y^{ \pm}=\left(W_{m}^{ \pm}, \rho^{ \pm}\right)^{T}, Y^{0}=\left(A, Z, \rho^{0}\right)^{T}$. Note that we use $V_{\mu}^{a}$ to denote the vector resonance components in the 
flavor basis and $\rho_{\mu}^{a}$ for the vector resonance components in the mass basis. ${ }^{4}$

In the limit $M_{W^{ \pm}}, M_{Z} \ll M_{\rho^{0}}$, equivalent to the condition $g, G \ll \sqrt{\alpha} g^{\prime \prime}$, the mixing matrices read

$$
\begin{aligned}
O^{(C)} & =\left(\begin{array}{cc}
1 & -g / g^{\prime \prime} \\
g / g^{\prime \prime} & 1
\end{array}\right), \\
O^{(N)} & =\left(\begin{array}{ccc}
g^{\prime} / G & g / G & -g / g^{\prime \prime} \\
g / G & -g^{\prime} / G & -g^{\prime} / g^{\prime \prime} \\
2 \frac{g g^{\prime}}{G g^{\prime \prime}} & \frac{g^{2}-g^{\prime 2}}{G g^{\prime \prime}} & 1
\end{array}\right) .
\end{aligned}
$$

In the same limit, the next-to-leading order approximations of the gauge-boson masses read

$$
\begin{aligned}
M_{W^{ \pm}} & =\frac{v g}{2}\left(1-\frac{g^{2}}{2 g^{\prime 2}}\right), \\
M_{\rho^{ \pm}} & =\frac{\sqrt{\alpha} v g^{\prime \prime}}{2}\left(1+\frac{g^{2}}{2 g^{\prime \prime 2}}\right),
\end{aligned}
$$

and

$$
\begin{aligned}
& M_{Z}=\frac{v G}{2}\left[1-\frac{\left(g^{2}-g^{\prime 2}\right)^{2}}{2 g^{\prime \prime 2} G^{2}}\right], \\
& M_{\rho^{0}}=\frac{\sqrt{\alpha} v g^{\prime \prime}}{2}\left(1+\frac{G^{2}}{2 g^{\prime \prime 2}}\right) .
\end{aligned}
$$

The leading order approximation for the partial width of the $\rho$ decay to the EW gauge bosons is

$$
\Gamma\left(\rho^{0} \rightarrow W^{+} W^{-}\right)=\Gamma\left(\rho^{ \pm} \rightarrow W^{ \pm} Z\right)=\frac{M_{\rho}^{5}}{48 \pi v^{4} g^{\prime \prime 2}} .
$$

The interactions of the gauge bosons with the Higgs can be read off from $\mathcal{L}_{\mathrm{hV}}$ in the mass basis

$$
\begin{aligned}
\frac{2 a_{V}}{v} \mathcal{M}(\alpha r) h=\frac{2 h}{v}[ & \frac{1}{2} c_{Z} M_{Z}^{2} Z_{\mu} Z^{\mu}+c_{W} M_{W}^{2} W_{\mu}^{+} W^{-\mu} \\
& +\frac{1}{2} c_{\rho^{0}} M_{\rho^{0}}^{2} \rho_{\mu}^{0} \rho^{0 \mu}+c_{\rho^{ \pm}} M_{\rho^{ \pm}}^{2} \rho_{\mu}^{+} \rho^{-\mu} \\
& +c_{W \rho^{ \pm}} M_{W} M_{\rho^{ \pm}}\left(W_{\mu}^{+} \rho^{-\mu}+\text { h.c. }\right) \\
& \left.+c_{Z \rho^{0}} M_{Z} M_{\rho^{0}} Z_{\mu} \rho^{0 \mu}\right]
\end{aligned}
$$

with the anomalousness factors ${ }^{5} c_{Y Y^{\prime}}$. If the flavor basis splitting factor $r$ equals $1\left(a_{\rho}=a_{V}\right)$ then the mass basis couplings $c_{Y Y^{\prime}}$ follow a simple pattern

$c_{Z}=c_{W}=c_{\rho^{0}}=c_{\rho^{ \pm}}=a_{V}, c_{Z \rho^{0}}=c_{W \rho^{ \pm}}=0$.

\footnotetext{
${ }^{4}$ We also used the subscripts $m$ and $f$ to distinguish the components of $W^{ \pm}$fields in the two bases. We do not use the subscripts if the choice of the basis is obvious from the context.

5 If $Y=Y^{\prime}$ then a single letter subscript will be used, e.g. $c_{Y Y} \rightarrow c_{Y}$.
}

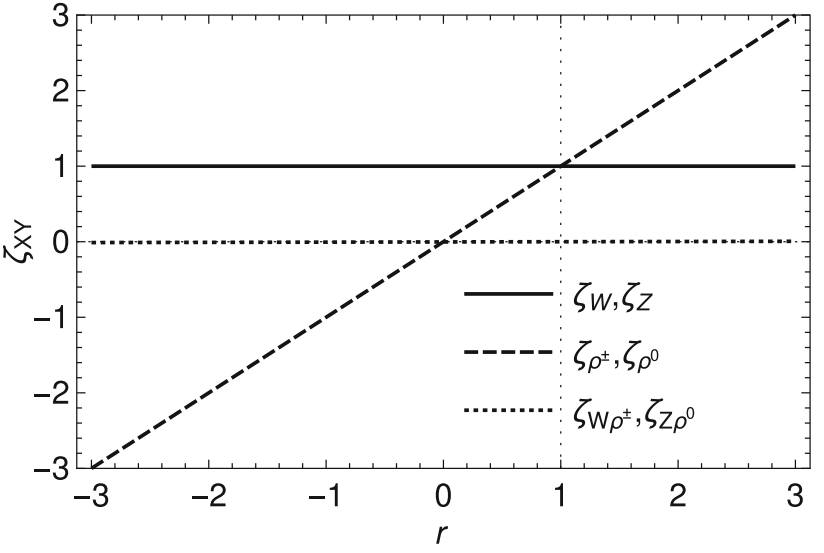

Fig. 1 The mass basis splitting factors $\zeta_{X Y}$ as functions of the splitting $r=a_{\rho} / a_{V}$. The graphs are plotted for $M_{\rho^{0}}=1.5 \mathrm{TeV}$ and $g^{\prime \prime}=15$

For a more general situation, $a_{\rho} \neq a_{V}$, the relations of $c_{Y Y^{\prime}}$ 's to $a_{V}$ and $a_{\rho}$ become more intricate. Let us introduce the mass basis splitting factors $\zeta_{Y Y^{\prime}}$ such that

$c_{Y Y^{\prime}}=a_{V} \zeta_{Y Y^{\prime}}$

Then

$\zeta_{Y Y^{\prime}}(\alpha, r)=\frac{O_{Y_{1} Y}(\alpha) \cdot\left[\boldsymbol{M}_{\mathbf{C}, \mathbf{N}}^{\mathbf{2}}(\alpha r)\right]_{Y_{1} Y_{2}} \cdot O_{Y_{2} Y^{\prime}}(\alpha)}{M_{Y}(\alpha) M_{Y^{\prime}}(\alpha)}$

The $\zeta_{Y Y^{\prime}}$ factors for the individual gauge bosons are summarized in Table 1 . There, we have introduced auxiliary variables

$\sqrt{D_{N}}=\frac{4}{v^{2}}\left(M_{\rho^{0}}^{2}-M_{Z}^{2}\right)$,

$\sqrt{D_{C}}=\frac{4}{v^{2}}\left(M_{\rho^{ \pm}}^{2}-M_{W}^{2}\right)$,

and

$k_{N}=\left[1-4 \alpha\left(g^{2}-g^{\prime 2}\right)^{2} / D_{N}\right]^{1 / 2}$,

$k_{C}=\left(1-4 \alpha g^{4} / D_{C}\right)^{1 / 2}$.

Note that $k_{N, C}=1-\mathcal{O}\left(x^{4}\right)$ where $x=g /\left(\sqrt{\alpha} g^{\prime \prime}\right) \approx$ $M_{W} / M_{\rho}$.

Figure 1 helps to understand how the flavor basis splitting, $r=a_{\rho} / a_{V}$, translates into the anomalous factors $c_{Y Y^{\prime}}$. The essential role in this issue is played by the $\zeta$ factors. Therefore, the graphs in the figure depict the dependences of $\zeta$ 's on $r$. There, the plots of $\zeta_{W, Z}$ are almost perfect horizontal lines at 1 which complies with $\zeta_{W, Z}=1-(1-r) \mathcal{O}\left(x^{4}\right)$. On the other hand, $\zeta_{\rho^{ \pm}, \rho^{0}}=r+(1-r) \mathcal{O}\left(x^{4}\right)$ suggests the straight line of the 45 degree slope for $\zeta_{\rho^{ \pm}, \rho^{0}}(r)$. Finally, the $\zeta$ factors of the mixed interaction terms are negligible when compared to the other $\zeta$ 's, which is in agreement with the finding that $\zeta_{W \rho^{ \pm}, Z \rho^{0}}=(1-r) \mathcal{O}\left(x^{2}\right)$.

While all $\zeta$ 's depend on $g^{\prime \prime}$ and $M_{\rho}$ these dependences are very weak. When changing $\left(g^{\prime \prime}, M_{\rho}\right)$ from $(10,1 \mathrm{TeV})$ 
Table 1 The mass basis splitting factors $\zeta_{Y Y^{\prime}}(\alpha, r)$ for the individual gauge bosons $Y$, $Y^{\prime} \in\left\{W^{ \pm}, \rho^{ \pm}, A, Z, \rho^{0}\right\}$

\begin{tabular}{llllll}
\hline$\zeta_{Y Y^{\prime}}(\alpha, r)$ & $\rho^{0}$ & $Z$ & $A$ & $\rho^{ \pm}$ & $W^{ \pm}$ \\
\hline$W^{ \pm}$ & 0 & 0 & 0 & $g^{2} \sqrt{\frac{\alpha}{D_{C}}}(r-1)$ & $\frac{1+r+(1-r) k_{C}}{2}$ \\
$\rho^{ \pm}$ & 0 & 0 & 0 & $\frac{1+r-(1-r) k_{C}}{2}$ & \\
$A$ & 0 & 0 & 0 & & \\
$Z$ & $\left(g^{2}-g^{\prime 2}\right) \sqrt{\frac{\alpha}{D_{N}}}(r-1)$ & $\frac{1+r+(1-r) k_{N}}{2}$ & & \\
$\rho^{0}$ & $\frac{1+r-(1-r) k_{N}}{2}$ & & & & \\
\hline
\end{tabular}
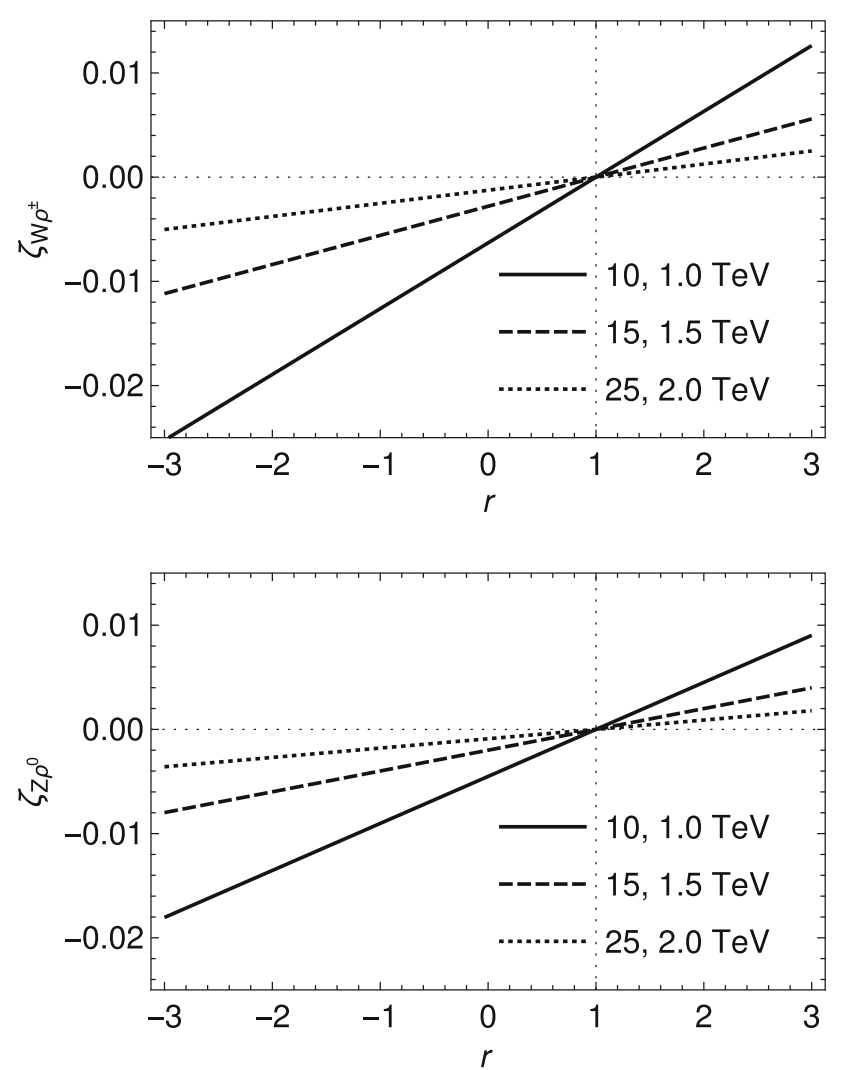

Fig. 2 The non-diagonal splitting factors $\zeta_{W \rho^{ \pm}}$(left panel) and $\zeta_{Z \rho^{0}}$ (right panel) as functions of the splitting $r=a_{\rho} / a_{V}$. The solid lines correspond to $\left(g^{\prime \prime}, M_{\rho}\right)=(10,1 \mathrm{TeV})$, the dashed lines to $(15,1.5 \mathrm{TeV})$, and the dotted lines to $(25,2 \mathrm{TeV})$

to $(25,2 \mathrm{TeV}), \zeta_{W}$ and $\zeta_{Z}$ vary no more than by about $10^{-4}$ for $-3 \leq r \leq 3$. The same conclusion applies to $\zeta_{\rho^{ \pm}}$and $\zeta_{\rho^{0}}$. The actual size of $\zeta_{W \rho^{ \pm}}$and $\zeta_{Z \rho^{0}}$ as well as their dependence on $g^{\prime \prime}$ and $M_{\rho}$ can be seen in Fig. 2. Consequently, for many phenomenological considerations the relations

$c_{Z}=c_{W}=a_{V}, \quad c_{\rho^{0}}=c_{\rho^{ \pm}}=a_{\rho}=r a_{V}$,

$c_{Z \rho^{0}}=c_{W \rho^{ \pm}}=0$,

represent satisfactory approximations over quite a large region of $r$ values and for all relevant values of $g^{\prime \prime}$ and $M_{\rho}$.

Now, let us turn our attention to the Higgs-to-gauge-boson couplings. Beside being proportional to the splitting factors the couplings are amplified by the (squares of) gauge-boson masses. Namely,

$g_{h W W}=a_{V} \zeta_{W} M_{W}^{2}, \quad g_{h Z Z}=a_{V} \zeta_{Z} M_{Z}^{2} / 2$,

$g_{h \rho^{+} \rho^{-}}=a_{V} \zeta_{\rho^{ \pm}} M_{\rho^{ \pm}}^{2}, \quad g_{h \rho^{0} \rho^{0}}=a_{V} \zeta_{\rho^{0}} M_{\rho^{0}}^{2} / 2$,

$g_{h W \rho^{ \pm}}=a_{V} \zeta_{W \rho^{ \pm}} M_{W} M_{\rho^{ \pm}}, \quad g_{h Z \rho^{0}}=a_{V} \zeta_{Z \rho^{0}} M_{Z} M_{\rho^{0}}$.

Then

$\frac{g_{h W W}}{g_{h Z Z}}=2 \frac{\zeta_{W}}{\zeta_{Z}} \frac{M_{W}^{2}}{M_{Z}^{2}}=2\left[1+(r-1) \mathcal{O}\left(x^{4}\right)\right] \frac{M_{W}^{2}}{M_{Z}^{2}}$.

Here, the whole dependence on new physics is contained in the $\mathcal{O}\left(x^{4}\right)$ term. Thus, the splitting can modify the SM expectation for the ratio (30) only very slightly. On the other hand, owing to the new vector triplet's large mass new physics becomes manifest the most in the Higgs interaction with the new vector triplet as can be seen in

$$
\begin{gathered}
\frac{g_{h \rho^{+} \rho^{-}}}{g_{h W W}}=\frac{\zeta_{\rho^{ \pm}}}{\zeta_{W}} \frac{M_{\rho^{ \pm}}^{2}}{M_{W}^{2}} \approx r \frac{M_{\rho^{ \pm}}^{2}}{M_{W}^{2}}, \\
\frac{g_{h \rho^{0} \rho^{0}}}{g_{h Z Z}}=\frac{\zeta_{\rho^{0}}}{\zeta_{Z}} \frac{M_{\rho^{0}}^{2}}{M_{Z}^{2}} \approx r \frac{M_{\rho^{0}}^{2}}{M_{Z}^{2}} .
\end{gathered}
$$

Note that while the vector mass affects the ratios significantly their dependences on $g^{\prime \prime}$ are completely ignorable. In Fig. 3, the ratios $g_{h \rho^{+} \rho^{-}} / g_{h W W}$ and $g_{h \rho^{0} \rho^{0}} / g_{h Z Z}$ as functions of $r$ and for various vector resonance masses are depicted.

If the splitting takes place new physics also manifests via the emergence of two new vertices, $h W^{ \pm} \rho^{ \pm}$and $h Z \rho^{0}$, not present either in the SM or in the Lagrangian $\mathcal{L}_{\mathrm{hV}}$ when $a_{\rho}=$ $a_{V}$. Even though the new coupling strengths lag far behind the strengths of the $h \rho^{+} \rho^{-}$and $h \rho^{0} \rho^{0}$ couplings their presence would introduce new phenomena. The relative strengths of the $h W^{ \pm} \rho^{ \pm}$and $h Z \rho^{0}$ couplings with respect to the $h W W$ and $h Z Z$ couplings, respectively, are given by 

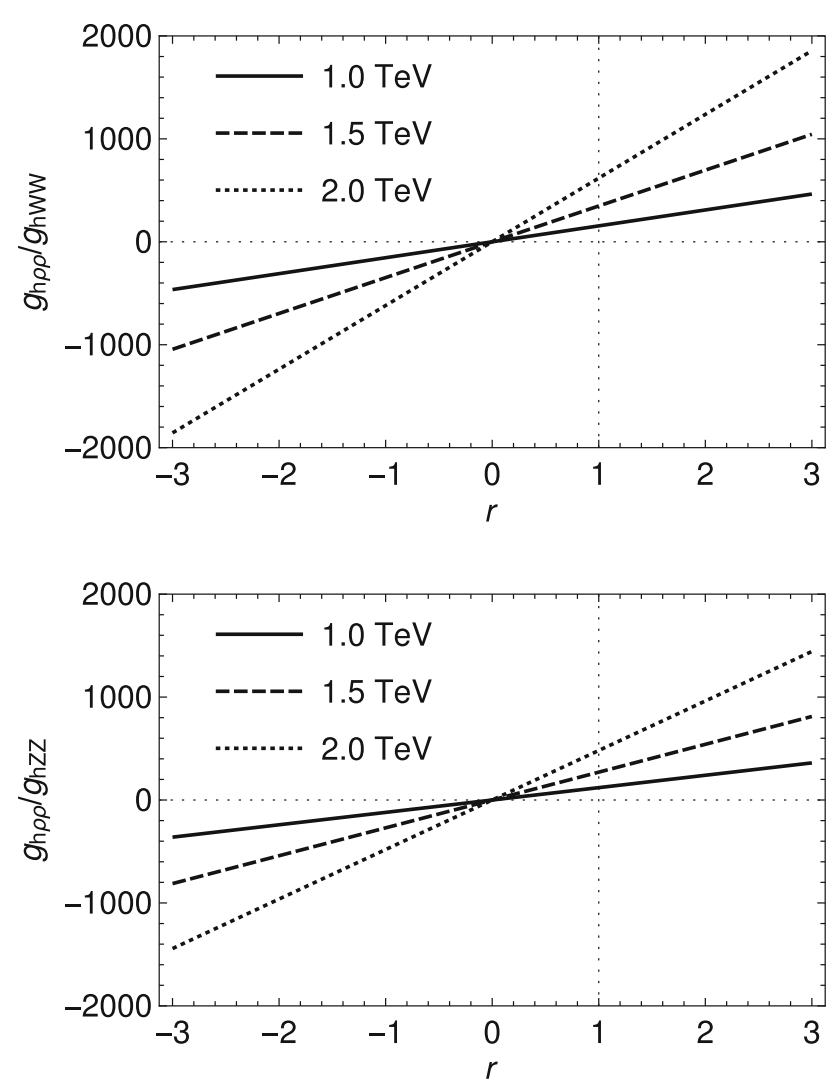

Fig. 3 The relative strengths of the $h \rho^{+} \rho^{-}$and $h \rho^{0} \rho^{0}$ couplings with respect to the $h W^{+} W^{-}$and $h Z Z$ couplings, respectively, as functions of the splitting $r=a_{\rho} / a_{V}$. The solid lines correspond to $M_{\rho}=1 \mathrm{TeV}$, the dashed lines to $1.5 \mathrm{TeV}$, and the dotted lines to $2 \mathrm{TeV}$

$$
\begin{aligned}
\frac{g_{h W \rho}}{g_{h W W}} & =\frac{\zeta_{W \rho}}{\zeta_{W}} \frac{M_{\rho^{ \pm}}}{M_{W}}=(r-1) \mathcal{O}(x), \\
\frac{g_{h Z \rho}}{g_{h Z Z}} & =2 \frac{\zeta_{Z \rho}}{\zeta_{Z}} \frac{M_{\rho^{0}}}{M_{Z}}=(r-1) \mathcal{O}(x) .
\end{aligned}
$$

Note that while these ratios are affected significantly by $g^{\prime \prime}$ their dependences on the vector mass are completely ignorable. In Fig. 4, the ratios $g_{h W \rho^{ \pm}} / g_{h W W}$ and $g_{h Z \rho^{0}} / g_{h Z Z}$ as functions of $r$ and for various $g^{\prime \prime}$ values are depicted.

The splitting of $a_{\rho}$ from $a_{V}$ will also cause $c_{W} \neq c_{Z}$. Thus, in principle, $r$ affects the model prediction for the custodial symmetry protected rho parameter. Fortunately, the effect is negligible for a wide range of $r$ values. To demonstrate it we inspect the prediction for the ratio $\lambda_{W Z} \equiv c_{W} / c_{Z}$

$\lambda_{W Z}=\frac{\zeta_{W}}{\zeta_{Z}}=1+(r-1) \mathcal{O}\left(x^{4}\right)$.

Numerically, our model predicts $\left|\lambda_{W Z}-1\right|<6 \times 10^{-5}$ at tree level when $-3 \leq r \leq 3,10 \leq g^{\prime \prime} \leq 25$, and $M_{\rho} \in(1,2) \mathrm{TeV}$. The current experimental limit is $\lambda_{W Z}=$ $0.88_{-0.09}^{+0.10}$ [39].
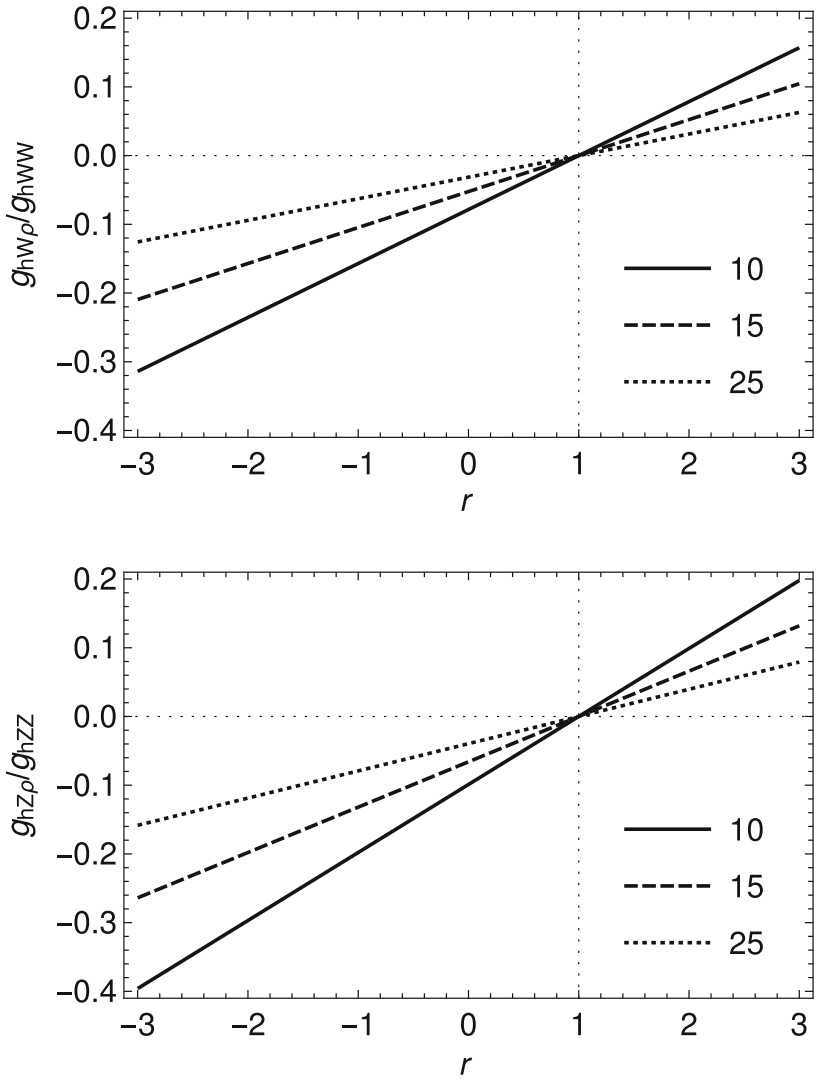

Fig. 4 The relative strengths of the $h W \rho^{ \pm}$and $h Z \rho^{0}$ couplings with respect to the $h W^{+} W^{-}$and $h Z Z$ couplings, respectively, as functions of the splitting $r=a_{\rho} / a_{V}$. The solid lines correspond to $g^{\prime \prime}=10$, the dashed lines to $g^{\prime \prime}=15$, and the dotted lines to $g^{\prime \prime}=25$

\section{Limits on the Higgs-related parameters}

\subsection{Relevant measurements}

In this paper, we would like to identify the restrictions that the current LHC measurements provide for the free parameters of the effective Lagrangian under consideration. While the LHC cannot compete yet with the low-energy data from the LEP, SLC, and Tevatron in setting a limit on the value of $g^{\prime \prime}$, it certainly plays the key role in restricting the Higgs-related couplings of the EW gauge bosons, fermions, and the vector resonance.

In [35], we calculated the indirect limits on $g^{\prime \prime}$ and other free parameters of the tBESS phenomenological Lagrangian, a special case of the Lagrangian considered here, fitting the observables $\epsilon_{1}, \epsilon_{2}, \epsilon_{3}, \Gamma_{b}(Z \rightarrow b \bar{b}+X)$, and $\mathrm{BR}\left(B \rightarrow X_{s} \gamma\right)$ under the assumption $a_{V}=a_{\rho}=1$ and $M_{\rho}=1 \mathrm{TeV}$ and $2 \mathrm{TeV}$. In addition to the setup investigated here, the tBESS model contains three independent direct interactions of the vector triplet with fermions: one with the left top- 
bottom quark doublet, another with the right top quark, and yet another with the right bottom quark. The analysis of the limits lead to the conclusion about the preference of higher $g^{\prime \prime}$ values, namely $g^{\prime \prime}>12$ at $95 \% \mathrm{CL}$ when combined with preferred values of other free parameters. Further, the analysis seemed to support the assumption of some models of partial fermion compositeness that the new strong physics resonances couple stronger to the right top quark than to the right bottom quark.

The requirement that our Lagrangian be treatable perturbatively bounds the values of $g^{\prime \prime}$ from above by the naive perturbativity limit, $g^{\prime \prime} / 2 \lesssim 4 \pi$, implying $g^{\prime \prime} \lesssim 25$. If we took this value as the final say in this issue it would not be reasonable to use $g^{\prime \prime}$ higher than about 20 in our calculations. However, one can imagine that a more rigorous analysis of the perturbativity limit could somehow modify its value one way or the other.

The limits on the Higgs-related couplings of the studied Lagrangian can be obtained from the existing measurements of the LHC experiments. In this paper, we use constraints from the ATLAS+CMS Collaborations analysis [39] based on the combination of six decay channels, namely $H \rightarrow \gamma \gamma$, $Z Z^{*}, W W^{*}, b \bar{b}, \tau \bar{\tau}$ and $\mu \bar{\mu}$, and of five production processes, namely gluon and vector boson fusions, and associated productions with $W, Z$, or a pair of top quarks. In our analysis, we assume that there is only one SM-like Higgs boson state at about $125 \mathrm{GeV}$ of a negligible decay width.

\subsubsection{The interim framework}

In [39], the combined ATLAS and CMS measurements were utilized to calculate fits and limits on the free kappa parameters of the interim framework introduced in [40]. In this framework, kappas parameterize possible deviations of the Higgs interactions from their SM expectations. The kappas are introduced so that the squared free parameters $\kappa_{i}$ scale the SM Higgs production cross sections $\sigma_{i}^{\mathrm{SM}}$ and/or the partial Higgs decay widths $\Gamma_{i}^{\mathrm{SM}}$ associated with the $i$ th SM particle. When $\kappa_{i}=1$ then the best available SM predictions for $\sigma_{i} \cdot \mathrm{BR}_{i}$ are recovered so that for the true SM Higgs boson no artificial deviations caused by ignored higher-order corrections are present.

The particular interim framework scenario that suits our effective Lagrangian is that of Section 6.1 of [39]. In this scenario, it is assumed that there are no non-SM decays of the Higgs and that the branching ratio of invisible and/or undetected decay products is zero. New particles in loops are allowed. Assuming that $\kappa_{c}=\kappa_{t}, \kappa_{s}=\kappa_{b}$, and $\kappa_{\mu}=\kappa_{\tau}$ there are generally seven free parameters in this scenario: five tree-level kappas $\left(\kappa_{W}, \kappa_{Z}, \kappa_{t}, \kappa_{b}, \kappa_{\tau}\right)$ and two loop-level kappas $\left(\kappa_{g}\right.$ and $\kappa_{\gamma}$ ). The loop-level kappas are associated with the higher-order effective vertices $H g g$ and $H \gamma \gamma$.
Table 2 The experimental limits for $\kappa$ 's that will be fitted to find the restrictions and the best values of $a_{V}, a_{\rho}$, and $c_{t}$. Note that the kappa limits were obtained under the assumption that $\kappa_{t}>0$

\begin{tabular}{lc}
\hline Parameter & ATLAS + CMS Limits \\
\hline$\kappa_{W}$ & $0.87 \pm 0.13$ \\
$\kappa_{Z}$ & $-0.98 \pm 0.10$ \\
$\kappa_{t}$ & $1.40 \pm 0.24$ \\
$\left|\kappa_{\gamma}\right|$ & $0.87 \pm 0.14$ \\
$\left|\kappa_{g}\right|$ & $0.78 \pm 0.13$ \\
\hline
\end{tabular}

We will derive the limits on $a_{V}, a_{\rho}$, and $c_{t}$ fitting the experimental values of kappas from Table 17 of [39] (the $B_{\mathrm{BSM}}=0$ part). The kappa values were obtained assuming $\kappa_{t}>0$ and using the following input parameters for the calculation of the SM quantities ${ }^{6}$ [41]: $M_{W}=80.398 \mathrm{GeV}$, $M_{Z}=91.1876 \mathrm{GeV}, G_{F}=1.16637 \times 10^{-5} \mathrm{GeV}^{-2}$, $M_{t}=172.5 \mathrm{GeV}, M_{h}=125.5 \mathrm{GeV}$. There are five kappas relevant for calculations of our limits: $\kappa_{\gamma}, \kappa_{W}, \kappa_{Z}, \kappa_{g}$, and $\kappa_{t}$. The remaining kappas, $\kappa_{b}$ and $\kappa_{\tau}$ do not depend on $a_{V}$, $a_{\rho}$, or $c_{t}$, at leading order.

The experimental uncertainties on kappas quoted in [39] are asymmetric. In our analysis, we will simplify the situation by equalizing both sides of the uncertainty to the one that is bigger. In addition, the 1-sigma interval of $\kappa_{Z}$ consists of two disconnected regions. In this case, we take into account only the region where the best-fit value of $\kappa_{Z}$ is placed. Thus modified experimental limits on $\kappa$ 's that will be used in our calculations are shown in Table 2.

\subsubsection{Fitting kappas with the effective Lagrangian parameters}

To restrict the free Higgs parameters via the fit to kappas the relations between the related kappas and $c_{i}$ 's have to be established. For that purpose we use some of the kappa defining quantities utilized in fitting the experiment and equate them to the predictions of our effective theory. With the input parameters listed above we obtain

$$
\begin{aligned}
\kappa_{W}^{2} & \equiv \frac{\Gamma_{W W^{*}}}{\Gamma_{W W^{*}}^{\mathrm{SM}}}=c_{W}^{2}, \quad \kappa_{Z}^{2} \equiv \frac{\Gamma_{Z Z^{*}}}{\Gamma_{Z Z^{*}}^{\mathrm{SM}}}=c_{Z}^{2}, \\
\kappa_{t}^{2} & \equiv \frac{\sigma_{t \bar{t} H}}{\sigma_{t \bar{t} H}^{\mathrm{SM}}}=c_{t}^{2}, \quad \kappa_{g}^{2} \equiv \frac{\sigma_{\mathrm{ggF}}}{\sigma_{\mathrm{ggF}}^{\mathrm{SM}}}=c_{t}^{2},
\end{aligned}
$$

where $\sigma_{t \bar{t} H}$ is the cross section of associated production of the Higgs boson with a pair of top quarks, $\sigma_{\mathrm{ggF}}$ is the gluonfusion Higgs production cross section, and $\Gamma_{j j}$ 's are the partial Higgs decay widths to the dibosons, $j j=W W^{*}, Z Z^{*}$. The index "SM" denotes the SM values.

\footnotetext{
${ }^{6}$ Here we quote only the values of those input quantities that will also be needed as inputs in the calculations in this paper.
} 
As is well known, the $H \rightarrow \gamma \gamma$ decay in the SM occurs at the loop level only. In the SM, two dominant contributions originate from the Feynman diagrams with the $W$ boson and top quark loops. Beyond the SM the anomalous couplings of the Higgs boson to $W$ and top quark are parameterized by the factors $c_{W}$ and $c_{t}$, respectively. In addition, the $H \rightarrow \gamma \gamma$ decay can be modified by the extra diagram with the $\rho^{ \pm}$ resonance in the loop. Thus, our effective theory predicts

$\kappa_{\gamma}^{2} \equiv \frac{\Gamma_{\gamma \gamma}}{\Gamma_{\gamma \gamma}^{\mathrm{SM}}}=\left[\frac{\alpha_{\mathrm{EM}}\left(g^{\prime \prime}, M_{\rho}\right)}{\alpha_{\mathrm{EM}}^{\mathrm{SM}}}\right]^{2}\left[\frac{c_{\gamma}\left(c_{W}, c_{t}, c_{\rho^{ \pm}}\right)}{c_{\gamma}^{\mathrm{SM}}}\right]^{2}$,

where $c_{\gamma}^{\mathrm{SM}}$ is the SM coupling of the $H \gamma \gamma$ effective vertex and $c_{\gamma}\left(c_{W}, c_{t}, c_{\rho^{ \pm}}\right)$is its anomalous analog. Further, $\alpha_{\mathrm{EM}}$ is the electromagnetic coupling constant which, in the case of the effective Lagrangian and for the given set of the input parameters [41], depends on new physics. The leading order approximation that dominates the ratio of the squared $\alpha_{\mathrm{EM}}$ 's when $M_{\rho} \gg M_{Z}$ reads

$$
\frac{\alpha_{\mathrm{EM}}^{2}\left(g^{\prime \prime}\right)}{\left(\alpha_{\mathrm{EM}}^{\mathrm{SM}}\right)^{2}}=\frac{1}{\left[1+16 \sqrt{2} G_{F} M_{W}^{2}\left(1-M_{W}^{2} / M_{Z}^{2}\right) / g^{\prime \prime 2}\right]^{2}} .
$$

Numerically, when $g^{\prime \prime}$ varies between 10 and 25 then $\left(\alpha_{\mathrm{EM}} / \alpha_{\mathrm{EM}}^{\mathrm{SM}}\right)^{2}$ changes from 0.992 to 0.999 . Thus, this effect will be ignored in our further analysis. Also, note that in the leading order the ratio is not affected by the mass of the vector resonance.

The contribution of the $\rho^{ \pm}$resonance to $h \rightarrow \gamma \gamma$ mimics the contribution of $W^{ \pm}$; the only difference comes from different masses and couplings of the vector particles. Thus, considering the principal contributions only-from the top, $W$, and $\rho$ loops $-c_{\gamma}$ reads

$$
\begin{aligned}
c_{\gamma}= & \frac{1}{8}\left[c_{t} N_{C} q_{t}^{2} F_{\text {ferm }}\left(x_{t}\right)+c_{W} F_{\mathrm{vec}}\left(x_{W}\right)\right. \\
& \left.+c_{\rho^{ \pm}} F_{\mathrm{vec}}\left(x_{\rho}\right)\right],
\end{aligned}
$$

where $N_{C} q_{t}^{2}=4 / 3, x_{i}=4 M_{i}^{2} / M_{h}^{2}$, and

$$
\begin{aligned}
F_{\text {ferm }}(x) & =-2 x[1+(1-x) f(x)], \\
F_{\text {vec }}(x) & =2+3 x+3 x(2-x) f(x),
\end{aligned}
$$

where

$$
f(x)= \begin{cases}\arcsin ^{2}(1 / \sqrt{x}), & x \geq 1, \\ -\frac{1}{4}\left[\ln \left(\frac{1+\sqrt{1-x}}{1-\sqrt{1-x}}\right)-i \pi\right]^{2}, & x<1 .\end{cases}
$$

For the given input values we get $F_{\text {ferm }}\left(x_{t}\right)=-1.38$, $F_{\text {vec }}\left(x_{W}\right)=8.34, F_{\text {vec }}\left(x_{1 \mathrm{TeV}}\right)=7.01$, and $F_{\mathrm{vec}}\left(x_{2 \mathrm{TeV}}\right)=$ 7.00. Then

$$
\begin{aligned}
c_{\gamma}\left(M_{\rho}=1-2 \mathrm{TeV}\right)= & -0.23 c_{t}+1.04 c_{W}+0.88 c_{\rho^{ \pm}} \\
= & -0.23 c_{t}+a_{V}\left[1.04 \zeta_{W}(r)\right. \\
& \left.+0.88 \zeta_{\rho^{ \pm}}(r)\right] .
\end{aligned}
$$

Note that, for the displayed decimal places, the numerical coefficients in this formula are not sensitive to varying the $\rho$ mass between 1 and $2 \mathrm{TeV}$.

The SM value of $c_{\gamma}$ is obtained when $c_{t}=c_{W}=1$ and $c_{\rho^{ \pm}}=0: c_{\gamma}^{\mathrm{SM}}=0.81$.

The effective Lagrangian predictions for observables do not change under the simultaneous sign change of all $c_{i}$ parameters. Therefore, a sign of one of $c_{i}$ 's can be fixed without losing physically distinguishable configurations of theory. Owing to that we choose $c_{t}>0$ throughout the paper.

\subsection{Failure of the $a_{V}=a_{\rho}$ scenario}

Before performing the full fit on $\kappa_{\gamma}, \kappa_{W}, \kappa_{Z}, \kappa_{g}$, and $\kappa_{t}$ with three free parameters $a_{V}, a_{\rho}, c_{t}$ let us provide a simple demonstration that the no-splitting scenario, $a_{V}=a_{\rho}$, has a hard time to satisfy the experimental restrictions on the kappas under consideration. Applying (37) to this situation we obtain

$\left|\kappa_{\gamma}\right|=\left|2.37 a_{V}-0.28 c_{t}\right|$.

Using the experimental restriction on $\kappa_{\gamma}$ (see Table 2) the Eq. (44) results in the allowed region comprised of two parallel stripes crossing the $a_{V}-c_{t}$ plane as shown in Fig. 5. Let us recall that we work under the assumption $c_{t}>0$ which reduces the $a_{V}-c_{t}$ plane to a half-plane.

In addition, the graphs in Fig. 5 display rectangular intersections of the experimentally allowed regions for $a_{V}$ and $c_{t}$. The limit on $a_{V}$ is derived by fitting $\kappa_{W}^{2}$ and $\kappa_{Z}^{2}$ while taking into account the no-splitting relations $c_{W}=c_{Z}=a_{V}$. The relevant $\chi^{2}$-function consists of the last two terms of (46). The obtained fit reads $\left|a_{V}\right|=0.93 \pm 0.08$ where the absolute value originate in the fitting of squares of variables. Analogically, the $c_{t}$ constraint is obtained by fitting $\kappa_{t}^{2}$ and $\kappa_{g}^{2}$. The corresponding $\chi^{2}$-function contains the second and third terms of (46). The obtained best value of $\left|c_{t}\right|$ is $c_{t}=0.85_{-0.12}^{+0.11}$.

The graphs in Fig. 5 indicate a tension between the $h \rightarrow$ $\gamma \gamma$ limits and the combined $\kappa_{W}-\kappa_{Z}$ and $\kappa_{t}-\kappa_{g}$ restrictions in the no-splitting version of our effective Lagrangian. In Sect. 3.3, we will assess if and to what extent this tension can be removed by allowing $a_{V}$ and $a_{\rho}$ to become independent.

\subsection{Full fit analysis}

Assuming that $a_{V}$ and $a_{\rho}$ are free independent parameters of the effective Lagrangian under consideration, we calculate 


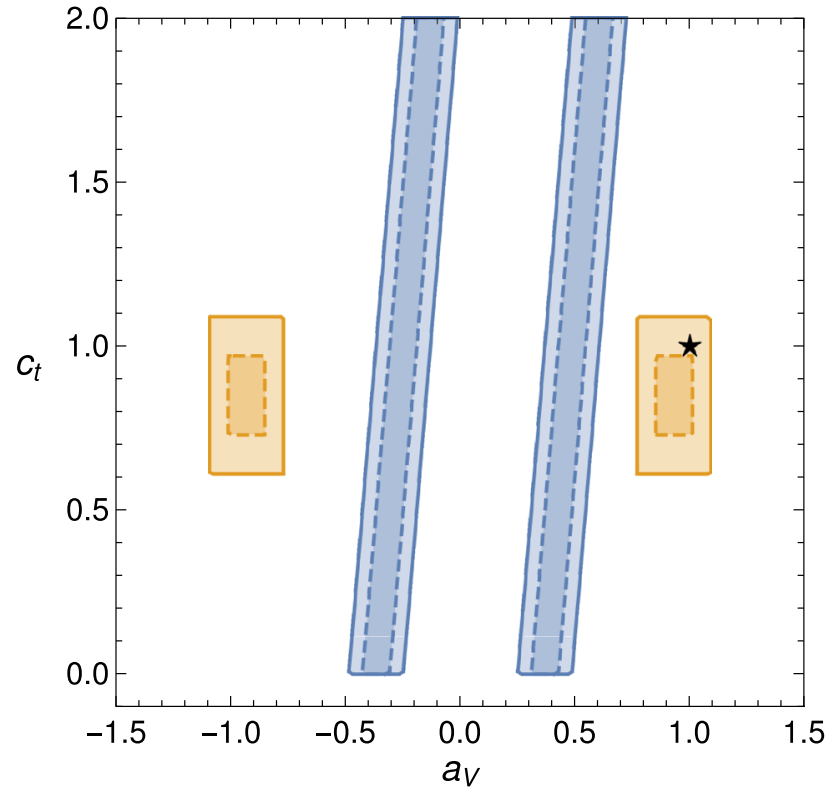

Fig. 5 The 1-sigma (dashed) and 2-sigma (solid) allowed regions (blue stripes) in the $a_{V}-c_{t}$ plane derived from the experimental restriction on $\kappa_{\gamma}$ under the no-splitting assumption. The orange rectangular areas correspond to the 1-sigma and 2-sigma allowed regions for $a_{V}$ and $c_{t}$ obtained by separate fitting of $\kappa_{W}^{2}, \kappa_{Z}^{2}$ and $\kappa_{t}^{2}, \kappa_{g}^{2}$, respectively. The star indicates the SM values

limits on $a_{V}, a_{\rho}$, and $c_{t}$ and search for their best values minimizing the $\chi^{2}$-function built out of the relevant measured observables and their predictions. We use the experimental values of $\kappa_{W}, \kappa_{Z}, \kappa_{t}, \kappa_{\gamma}$, and $\kappa_{g}$ shown in Table 2 . Theoretical predictions of the kappas in terms of $c_{Z}, c_{W}, c_{\rho^{ \pm}}$, and $c_{t}$ of the Lagrangian interaction term (18) and (5), respectively, are given in (36) and (37). The dependence of $c_{\gamma}$ on $c_{W}, c_{\rho^{ \pm}}$, and $c_{t}$ in (37) is given by (39). The $c_{Z}, c_{W}$, and $c_{\rho^{ \pm}}$are, in turn, related to $a_{V}$ and $a_{\rho}$ via (20).

In the interim framework the squares of kappas rather than the kappas themselves are defined and related to measured observables. Therefore, our theoretical predictions are also related to squared kappas and the best fit should be sought for minimizing the following $\chi^{2}$-function:

$$
\begin{aligned}
\chi^{2}\left(a_{V}, a_{\rho}, c_{t}\right)= & \left\{\frac{\kappa_{\gamma}^{2}-\left[c_{\gamma}\left(a_{V}, a_{\rho}, c_{t}\right) / c_{\gamma}^{\mathrm{SM}}\right]^{2}}{\tilde{\sigma}_{\gamma}}\right\}^{2} \\
& +\sum_{i=t, g}\left(\frac{\kappa_{i}^{2}-c_{t}^{2}}{\tilde{\sigma}_{i}}\right)^{2} \\
& +\sum_{i=W, Z}\left[\frac{\kappa_{i}^{2}-c_{i}^{2}\left(a_{V}, a_{\rho}\right)}{\tilde{\sigma}_{i}}\right]^{2},
\end{aligned}
$$

where $\tilde{\sigma}_{i}$ 's are the experimental errors for $\kappa_{i}^{2}$ 's. The fitting of squares introduces degeneracy of solutions caused by the insensitivity of $\chi^{2}$ to the relative signs between the kappas and theory parameters. Recall that $c_{t}>0$ by assumption.
If we approximate $c_{W}=c_{Z}=a_{V}$ and $c_{\rho}^{ \pm}=a_{\rho}$ the Eq. (45) can be simplified without any significant impact on the best-fit values as can be inferred from our conclusions obtained in Sect. 2 (viz., the Eq. (26)). Then, substituting (43) into (45) we obtain

$$
\begin{aligned}
\tilde{\chi}^{2}\left(a_{V}, a_{\rho}, c_{t}\right)= & {\left[\frac{\kappa_{\gamma}^{2}-\left(1.28 a_{V}+1.09 a_{\rho}-0.28 c_{t}\right)^{2}}{2 \kappa_{\gamma} \sigma_{\gamma}}\right]^{2} } \\
& +\left(\frac{\kappa_{t}^{2}-c_{t}^{2}}{2 \kappa_{t} \sigma_{t}}\right)^{2}+\left(\frac{\kappa_{g}^{2}-c_{t}^{2}}{2 \kappa_{g} \sigma_{g}}\right)^{2} \\
& +\left(\frac{\kappa_{W}^{2}-a_{V}^{2}}{2 \kappa_{W} \sigma_{W}}\right)^{2}+\left(\frac{\kappa_{Z}^{2}-a_{V}^{2}}{2 \kappa_{Z} \sigma_{Z}}\right)^{2}
\end{aligned}
$$

It is obvious that $\tilde{\chi}^{2}$ has degenerate minima. If the $\tilde{\chi}^{2}$ function assumes its minimum value $\tilde{\chi}_{\min }^{2}$ for some triplet $a_{V}, a_{\rho}, c_{t}$ then also $\tilde{\chi}^{2}\left(-a_{V},-a_{\rho},-c_{t}\right)=\tilde{\chi}_{\min }^{2}$. Nevertheless, by fixing $c_{t}>0$ we eliminate half of the degenerate minima.

A simple inspection of the Eq. (46) implies that $\tilde{\chi}^{2}$ can be minimized when, at the same time, $c_{t}$ assumes the value of "weighted average" of $\kappa_{t}$ and $\kappa_{g},\left|a_{V}\right|$ assumes a "weighted average" of $\kappa_{W}$ and $\kappa_{Z}$, and $a_{\rho}$ sets to zero the first term with the before-obtained values of $c_{t}$ and $a_{V}$ substituted in. Zeroing the first term of (46) amounts to solving a quadratic equation in $a_{\rho}$. Thus, there are generally two solutions for $\left(a_{V}, c_{t}\right)$ and two solutions for $\left(-a_{V}, c_{t}\right)$, i.e. four solutions in total. Since the first term of Eq. (46) has zero contribution to $\tilde{\chi}_{\min }^{2}$ and because the following two terms depend only on $c_{t}$ and two last terms only on $\left|a_{V}\right|$, all four solutions result in the same value of $\tilde{\chi}_{\min }^{2}$.

In particular, by fitting the kappa values of Table 2 we get four minimizing triplets of $\left\{a_{V}, a_{\rho}, c_{t}\right\}$ with the same minimal values of $\tilde{\chi}^{2}, \tilde{\chi}_{\min }^{2}=4.17$. Having d.o.f. $=5-3=$ 2 , the value corresponds to the hypothesis backing of $12 \%$. The values of the degenerate best-fit triplets along with the corresponding parameter constraints at 20,68, and 95\% CL are shown in Table 3.

The graphic representation of the best-fit values is depicted in Fig. 6. There, the two-dimensional cut of the $c_{t}-a_{\rho}-a_{V}$ allowed regions by the $c_{t}=0.85$ plane is shown. Note that 0.85 is the best-fit value of $c_{t}$. The contours depicted in the graph correspond to the 68 and $95 \% \mathrm{CL}$ regions in the $c_{t}-$ $a_{\rho}-a_{V}$ space. The splitting factors $r$ for the best-fit points $\mathrm{A}, \mathrm{B}, \mathrm{C}$, and D have the values $-0.09,-1.81,-0.56$, and -2.27 , respectively. The straight line indicates the points of the no-splitting scenario, $a_{\rho}=a_{V}$. The full 95\% CL region in the $c_{t}-a_{\rho}-a_{V}$ space around the best-fit point $\mathrm{A}$ is shown in Fig. 7. The allowed regions around the best-fit points $B$, $\mathrm{C}$, and $\mathrm{D}$ are very similar in shape and size to the region $\mathrm{A}$.

There is a good reason why $a_{V} a_{\rho}<0$ for all four best-fit points. It is because the combined contributions of $a_{V}$ and 
Table 3 The best-fit values of $c_{t}, a_{V}$, and $a_{\rho}$ corresponding to the four minima of the $\tilde{\chi}^{2}$-function (46) with the constraints at $20 \% \mathrm{CL}$ (1-sigma), 68\% CL, and 95\% CL. All minima (labeled as $\mathrm{A}, \mathrm{B}, \mathrm{C}$, and D) have the same backing of $12 \%$

\begin{tabular}{|c|c|c|c|c|c|c|c|c|}
\hline Parameter & \multicolumn{2}{|c|}{ A } & \multicolumn{2}{|c|}{ B } & \multicolumn{2}{|c|}{$\mathrm{C}$} & \multicolumn{2}{|c|}{$\mathrm{D}$} \\
\hline \multicolumn{9}{|l|}{$c_{t}$} \\
\hline $20 \% \mathrm{CL}$ & & $\begin{array}{l}+0.11 \\
-0.12\end{array}$ & & $\begin{array}{l}+0.11 \\
-0.12\end{array}$ & & $\begin{array}{l}+0.11 \\
-0.12\end{array}$ & & $\begin{array}{l}+0.11 \\
-0.12\end{array}$ \\
\hline $68 \% \mathrm{CL}$ & 0.85 & $\begin{array}{l}+0.19 \\
-0.25\end{array}$ & 0.85 & $\begin{array}{l}+0.19 \\
{ }_{-0.25}\end{array}$ & 0.85 & $\begin{array}{l}+0.19 \\
{ }_{-0.25}\end{array}$ & 0.85 & $\begin{array}{l}+0.19 \\
-0.25\end{array}$ \\
\hline $95 \% \mathrm{CL}$ & & $\begin{array}{l}+0.27 \\
-0.43\end{array}$ & & $\begin{array}{l}+0.27 \\
{ }_{-0.43}\end{array}$ & & $\begin{array}{l}+0.27 \\
-0.43\end{array}$ & & $\begin{array}{l}+0.27 \\
-0.43\end{array}$ \\
\hline \multicolumn{9}{|l|}{$a_{V}$} \\
\hline $20 \% \mathrm{CL}$ & & $\begin{array}{l}+0.08 \\
-0.08\end{array}$ & & $\begin{array}{l}+0.08 \\
-0.08\end{array}$ & & $\begin{array}{l}+0.08 \\
-0.08\end{array}$ & & $\begin{array}{l}+0.08 \\
-0.08\end{array}$ \\
\hline $68 \% \mathrm{CL}$ & 0.93 & $\begin{array}{l}+0.14 \\
-0.16\end{array}$ & 0.93 & $\begin{array}{l}+0.14 \\
-0.16\end{array}$ & -0.93 & $\begin{array}{l}+0.16 \\
{ }_{-0.14}\end{array}$ & -0.93 & $\begin{array}{l}+0.16 \\
{ }_{-0.14}\end{array}$ \\
\hline $95 \% \mathrm{CL}$ & & $\begin{array}{l}+0.20 \\
-0.25\end{array}$ & & $\begin{array}{l}+0.20 \\
+-0.25\end{array}$ & & $\begin{array}{l}+0.25 \\
{ }_{-0.20}\end{array}$ & & $\begin{array}{l}+0.25 \\
{ }_{-0.20}\end{array}$ \\
\hline \multicolumn{9}{|l|}{$a_{\rho}$} \\
\hline $20 \% \mathrm{CL}$ & & $\begin{array}{l}+0.16 \\
-0.17\end{array}$ & & $\begin{array}{l}+0.17 \\
-0.15\end{array}$ & & $\begin{array}{l}+0.17 \\
{ }_{-0.16}\end{array}$ & & $\begin{array}{l}+0.16 \\
-0.17\end{array}$ \\
\hline $68 \% \mathrm{CL}$ & -0.08 & $\begin{array}{l}+0.29 \\
-0.34\end{array}$ & -1.68 & $\begin{array}{l}+0.34 \\
-0.28\end{array}$ & 0.52 & $\begin{array}{l}+0.33 \\
-0.29\end{array}$ & 2.11 & $\begin{array}{l}+0.28 \\
-0.34\end{array}$ \\
\hline $95 \% \mathrm{CL}$ & & $\begin{array}{l}+0.43 \\
-0.58\end{array}$ & & $\begin{array}{l}+0.58 \\
-0.40\end{array}$ & & $\begin{array}{l}+0.58 \\
-0.44\end{array}$ & & $\begin{array}{l}+0.41 \\
-0.58\end{array}$ \\
\hline
\end{tabular}

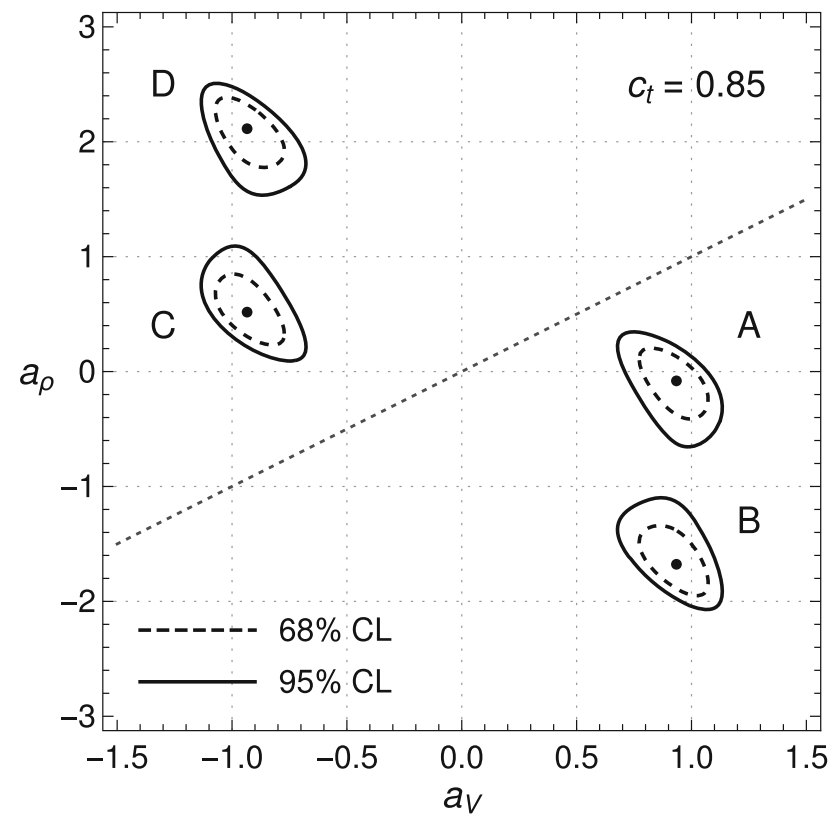

Fig. 6 The two-dimensional cut of the three-dimensional region of the allowed values of parameters $a_{V}, a_{\rho}$, and $c_{t}$ when $c_{t}$ is fixed at its best value of 0.85 . The dots indicate the best-fit values of the fit. They are labeled as $A, B, C$, and $D$ in correspondence with Table 3 . The dashed and solid contours show $68 \% \mathrm{CL}$ and $95 \% \mathrm{CL}$ limits. The straight line indicates the points of the no-splitting scenario, $a_{\rho}=a_{V}$

$c_{t}$ to $\left|c_{\gamma}\right|$ - the values of $a_{V}$ and $c_{t}$ being determined by the other terms of the $\chi^{2}$-function (46)-overshoot the optimal value of $\left|c_{\gamma}\right|$. Since the $a_{V}$ contribution dominates the $c_{t}$ contribution, the $a_{\rho}$ has to have a sign opposite to the sign of $a_{V}$ in order to counterbalance the excess. In addition, since we optimize $\left|c_{\gamma}\right|$, rather than $c_{\gamma}$ itself, $a_{\rho}$ resulting in the optimal $c_{\gamma}$ plays as well as $a_{\rho}$ resulting in $-c_{\gamma}$. Thus, we end up with two $a_{\rho}$ 's for each of the two best-fit values of $a_{V}$.

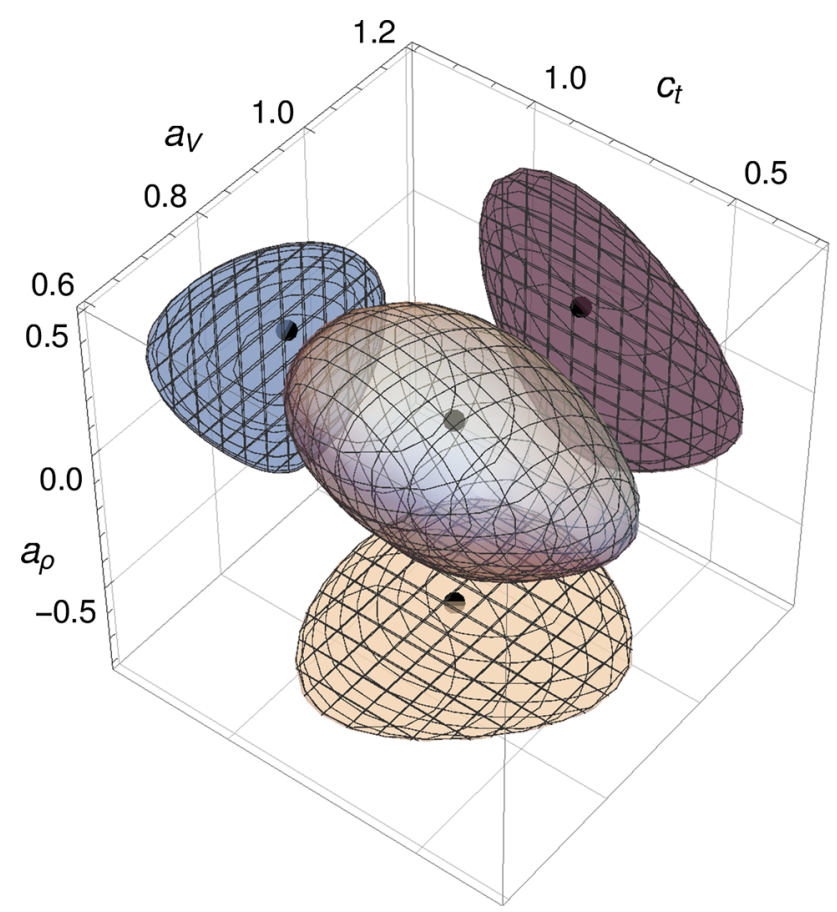

Fig. 7 The 95\% CL allowed region of the parameters $a_{V}, a_{\rho}$, and $c_{t}$ of the $\tilde{\chi}^{2}$ minima $\mathrm{A}$ and the region projections to two-parameter planes. Dots indicate the best-fit value and its projections to the planes

It is not a difficult exercise to impose the no-splitting condition, $a_{\rho}=a_{V}$, on $\tilde{\chi}^{2}$ of (46) in order to obtain a more rigorous quantification and justification of the conclusion we have reached in Sect. 3.2. The fitting of $\tilde{\chi}^{2}$ results in two minima of unequal depths. This asymmetry results from the sensitivity of $\tilde{\chi}^{2}$ on the relative sign of $a_{V}$ and $c_{t}$ in the $\kappa_{\gamma}$ term. The global minimum assumes the value $\tilde{\chi}_{\min }^{2}=21.3$ which for d.o.f. $=5-2=3$ corresponds to the hypothesis backing of $0.9 \%$. The minimum resides at $a_{V}=0.55 \pm 0.05$ and $c_{t}=0.89_{-0.11}^{+0.10}$. The local minimum 


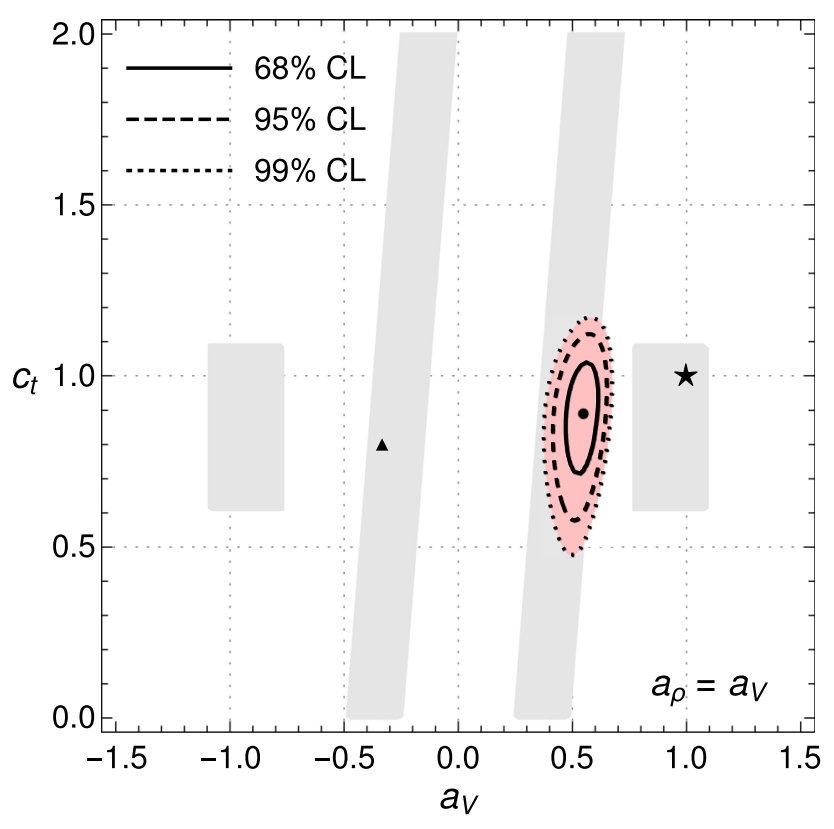

Fig. 8 The fit under the no-splitting condition and the related CL contours. The multiply encircled red area corresponds to the allowed regions of 68, 95, and 99\% CL. The dot inside the area indicates the best-fit value. The triangle indicates the position of the second (local) minimum of the $\chi^{2}$-function. The star indicates the SM values of the fitting parameters. For comparison, the graph is interlaced with the reminiscence of Fig. 5 (gray regions) where the partial limits obtained by a cruder approach were shown

at $\left(a_{V}, c_{t}\right)=(-0.33,0.81)$ amounts to the value higher by $\Delta \tilde{\chi}^{2}=10.7$ above the global minimum.

The fit under the no-splitting condition and the related CL contours are depicted in Fig. 8. Besides, the graph contains reminiscence of Fig. 5 where the limits obtained by the cruder approach of Sect. 3.2 were shown. From a naive visual inspection we would say that the results of the cruder analysis do not contradict the more sophisticated fit performed here. Not only the position of the minimum coincides with the guessed expectation based on Fig. 5, but the obtained hypothesis backing also confirms our qualitative conclusion of Sect. 3.2 about a low support of the data for the no-splitting scenario.

Having the rho-to-Higgs coupling disconnected from the Higgs interactions with the EW gauge bosons and acknowledging that $a_{V}=c_{t}=1$ is compatible with the experiment, one might wonder how successful the parameter $a_{\rho}$ alone would be in accommodating the existing data if $a_{V}$ and $c_{t}$ were kept on their SM values. This simple exercise amounts to minimizing the $\tilde{\chi}^{2}$-function (46) when $a_{V}=c_{t}=1$. There are two minima, at $a_{\rho}=-0.12$ and $a_{\rho}=-1.72$, both with $\chi_{\min }^{2}=7$. Since d.o.f. $=5-1=4$ our effective Lagrangian with the SM couplings of the Higgs boson to the EW gauge bosons and top quark has $13.8 \%$ backing by the data. Raising $\chi^{2}$ above its minimum by 1 the 1 -sigma
$(68 \% \mathrm{CL})$ constraints read ${ }_{-0.14}^{+0.12}$ and $_{-0.12}^{+0.14}$ for the former and latter $a_{\rho}$ best values, respectively. Hence, if we had a theoretical reason to demand $a_{V}=c_{t}=1$ the experimental support for the vector triplet of particular $a_{\rho}$ values would be as good as in the model with loose $a_{V}$ and $c_{t}$.

There are two kappas in the combined ATLAS+CMS measurements [39] not utilized in our analysis: $\kappa_{\tau} \equiv$ $\Gamma_{\tau^{+} \tau^{-}} / \Gamma_{\tau^{+} \tau^{-}}^{\mathrm{SM}}$ and $\kappa_{b} \equiv \Gamma_{b \bar{b}} / \Gamma_{b \bar{b}}^{\mathrm{SM}}$. In the approximation used in this paper, $\kappa_{\tau}$ is solely related to $c_{\tau}, \kappa_{\tau}^{2}=c_{\tau}^{2}$. Thus, it has no impact on the fits of other parameters. Regarding $\kappa_{b}$, while it shares the dependence on $c_{b}$ with $\kappa_{g}$, its effect on the Higgs production via gluon-gluon fusion is negligible. Consequently, we had dropped the $\kappa_{b}$ term from the $\chi^{2}$-function.

On the other hand, the effective coupling $\kappa_{Z \gamma} \equiv \Gamma_{Z \gamma} / \Gamma_{Z \gamma}^{\mathrm{SM}}$ has a similar structure as $\kappa_{\gamma}$ : it depends on $a_{V}, a_{\rho}$, and $c_{t}$ parameters at leading order. Thus, it has a potential to affect our fits significantly. Unfortunately, the existing measurements restrict $\kappa_{Z \gamma}$ very poorly [42]. Because of that, neither $\kappa_{Z \gamma}$ was included in the $\chi^{2}$-function (45).

\subsection{Unitarity limits for the preferred values of the Higgs couplings}

In this Section, we would like to determine how the usability of our phenomenological Lagrangian is restricted by the unitarity limits when the data preferred Higgs couplings obtained in Sect. 3.3 are considered.

Opposite to the SM, our effective Lagrangian is not renormalizable and its applicability is limited to a finite range of energies, not exceeding the point where scattering amplitudes violate unitarity. Considering the low-energy phenomenology of underlying new strong physics it is natural to demand that its successful effective description does not break down below the scale significant to these new interactions. Thus, the results of the investigation of the unitarity limits could be suggestive either of the new physics scale or, in a less fortunate case, of the defects in our Lagrangian. The least we can, and need, to deduce from such an analysis is the range of usability of the effective Lagrangian we play with.

The unitarity of our Lagrangian was investigated in detail in our previous publications $[34,35]$. Our analysis was based on the scattering amplitudes of the longitudinal EW gauge bosons to the EW gauge bosons. We studied the unitarity of the amplitudes using the Equivalence theorem approximation where the concerned amplitudes were replaced by the pionic scattering amplitudes of the non-linear sigma model. While the approximation corresponds to the limit $g, g^{\prime} \rightarrow 0$ (no pion to EW gauge-boson vertices), the exchange of the Higgs and vector resonances was included.

Even more thorough investigation of the unitarity limits of the same (in relevant sectors) effective Lagrangian was performed in [31]. Beside the elastic $\pi \pi \rightarrow \pi \pi$ ampli- 
tudes, authors of [31] analyzed the unitarity limits implied by the non-elastic $\pi \pi \rightarrow h h, \rho_{L} \rho_{L}, h \rho_{L}$ processes. Note that wherever applicable the conclusions of [31] coincide with our conclusions $[34,35]$. In the following, we utilize the findings of these papers for calculation of the tree-level unitarity limits for our Lagrangian. Skipping details of the very standard calculations, below we summarize and discuss the obtained results.

In Fig. 9, we show the $\pi \pi \rightarrow \pi \pi$ tree-level unitarity limits. ${ }^{7}$ In our case, the $\pi \pi \rightarrow \pi \pi$ scattering amplitudes depend only on one of the three Higgs couplings investigated in this paper, namely $\left|a_{V}\right|$. Besides, the amplitudes also depend on $M_{\rho}$ and $g^{\prime \prime}$. There are three graphs in Fig. 9 corresponding to three different masses of the vector resonance: $M_{\rho}=1,1.5$, and $2 \mathrm{TeV}$. In each graph, there are regions shown where unitarity holds up to $\Lambda=3 \mathrm{TeV}, 4 \mathrm{TeV}$, and $5 \mathrm{TeV}$. The regions are superimposed by the experimentally allowed 2-sigma interval for $a_{V}$ obtained in Sect. 3.3: $0.77 \leq a_{V} \leq 1.09$, the best fit being $a_{V}=0.93$.

We can see that for the $1 \mathrm{TeV}$ vector resonance unitarity holds up to at least $\Lambda=3 \mathrm{TeV}$ when $a_{V}$ and $g^{\prime \prime}$ assumes allowed values, ${ }^{8}$ excluding a small region where $g^{\prime \prime} \rightarrow 12$ and $a_{V} \rightarrow 1.1$. When raising $M_{\rho}$, the region where unitarity holds shrinks toward higher $g^{\prime \prime}$. At the same time, it slightly shifts toward smaller $a_{V}$.

If we dropped the vector resonance from our effective Lagrangian the Higgs resonance alone could unitarize the elastic $\pi \pi$ amplitudes up to some $\Lambda_{h}$ that depends on $a_{V}$; for example, if $a_{V}$ assumes its best value of 0.93 the treelevel unitarity holds up to $\Lambda_{h}=4.6 \mathrm{TeV}$. Perhaps, it might also be worth mentioning that $\Lambda_{h} \rightarrow \infty$ when $a_{V}=1$. One wonders how adding the vector triplet to the Higgs-only setup changes the $\Lambda_{h}$ limit. To assess it we plot the graph in Fig. 10. There, for given $a_{V}$ and $M_{\rho}$, the values of $g^{\prime \prime}$ are divided into two intervals: the one where $\Lambda<\Lambda_{h}\left(a_{V}\right)$, and the other, where $\Lambda>\Lambda_{h}\left(a_{V}\right)$. We can see in the graph that if $a_{V} \geq 1$ adding the vector resonance will always lower the unitarity limit. On the other hand, if $a_{V}<1$ there is always $g_{0}^{\prime \prime}$ such that for all $g^{\prime \prime} \geq g_{0}^{\prime \prime}$ the unitarity limit gets bigger.

In general, the $\pi \pi$ scattering amplitudes are plagued with the linear growths in $s$. Any added ingredient or assumption that removes the linear growths has a good chance to improve unitarity limits. It can be shown [31] that assuming special relations among the parameters of our Lagrangian (the sum rules) or adding new pseudo-scalar and/or axial-vector fields to our Lagrangian can eliminate the linear terms from the

\footnotetext{
7 All our calculations use the $\Gamma_{\rho}=0$ approximation. This is justifiable as long as $M_{\rho}$ is far below the unitarity limit in terms of the $\rho$ width.

8 Recall that $12 \leq g^{\prime \prime} \leq 8 \pi$.
}

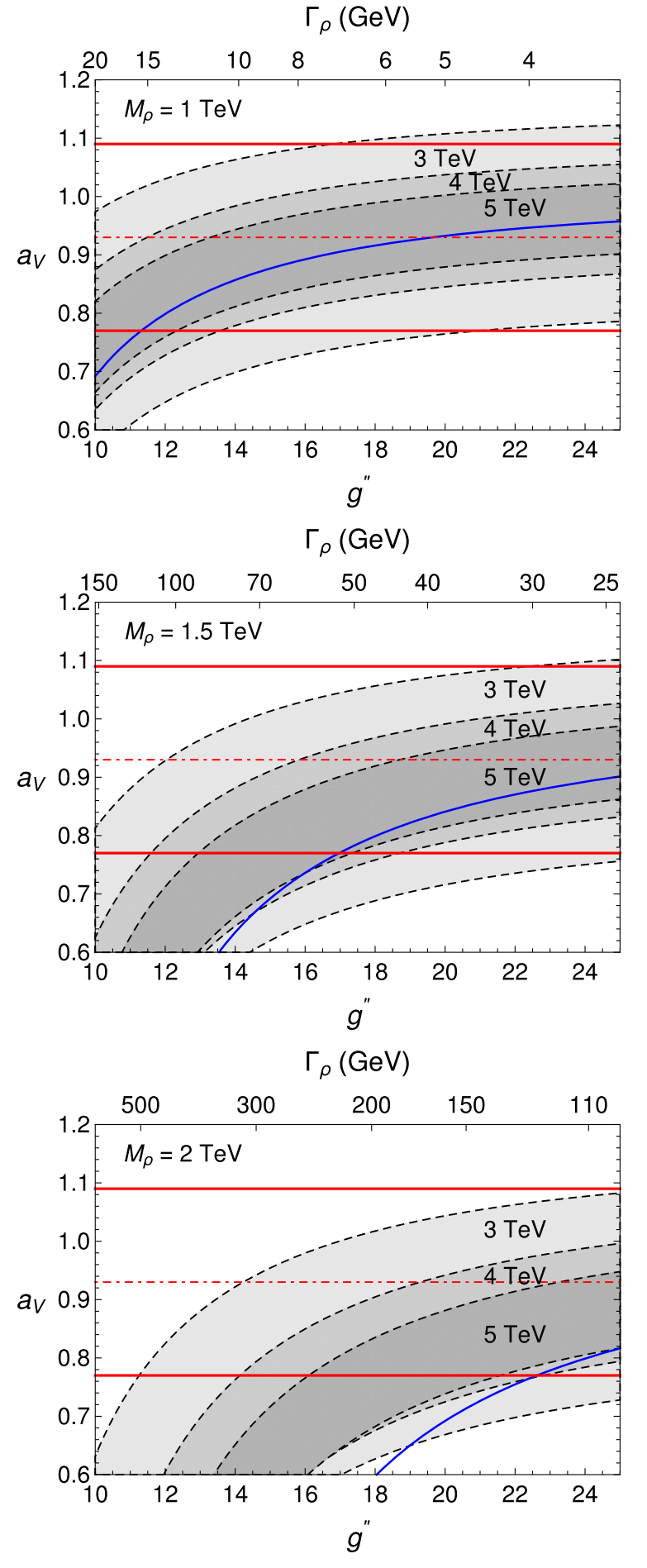

Fig. 9 The tree-level unitarity constraints from $\pi \pi \rightarrow \pi \pi$ in the $a_{V}-$ $g^{\prime \prime}$ plane for different masses of the vector triplet, $M_{\rho}=1,1.5$, and $2 \mathrm{TeV}$ in the clockwise direction. The shaded areas indicate regions where the unitarity holds, up to 3,4 , and $5 \mathrm{TeV}$. Two horizontal (solid red) lines show allowed interval of values of $a_{V}$ at 2-sigma, the dotdashed line is the best-fit value $a_{V}=0.93$. The blue solid curve plots the sum rule (47). The upper axis labels show the vector resonance decay widths corresponding to $g^{\prime \prime}$ 's of the bottom axis 


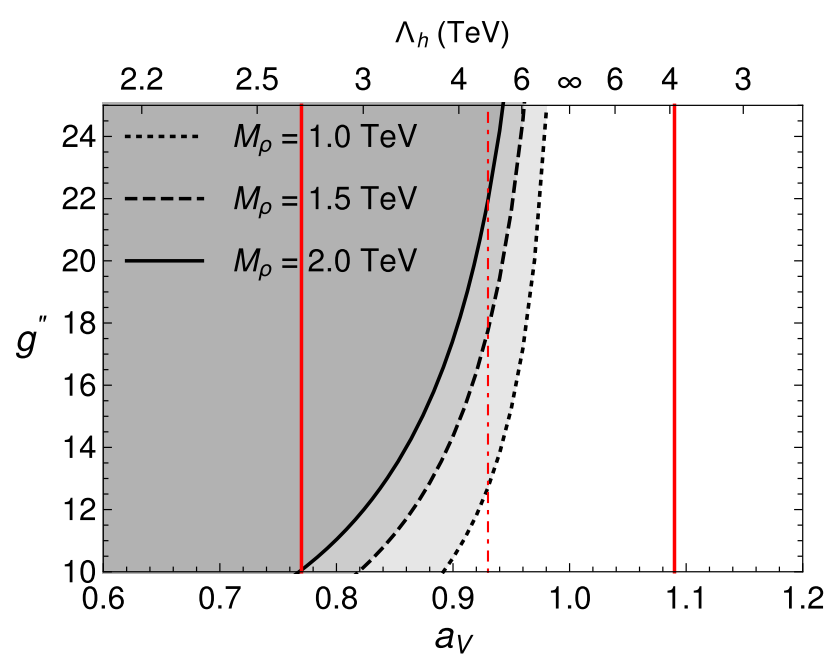

Fig. 10 The regions where the unitarity limit of our model $\left(a_{V}, g^{\prime \prime}, M_{\rho}\right)$ gets bigger (darker area) and smaller (lighter area) than the unitarity limit $\Lambda_{h}\left(a_{V}\right)$ of the Higgs-only model of a given value $a_{V}$. The regions are depicted for three different masses $M_{\rho}=1,1.5$, and $2 \mathrm{TeV}$. Two vertical (solid red) lines show allowed interval of values of $a_{V}$ at 2-sigma, the dot-dashed line is the best-fit value $a_{V}=0.93$

scattering amplitudes. For example, the relation

$a_{V}^{2}+\frac{3}{4} \alpha=1$

removes the linear dependence on $s$ from the $\pi \pi \rightarrow \pi \pi$ amplitude. The sum rule (47) links $a_{V}$ with $g^{\prime \prime}$ and $M_{\rho}$ via $\alpha=\left[2 M_{\rho} /\left(v g^{\prime \prime}\right)\right]^{2}$. The resulting sets of $\left(a_{V}, g^{\prime \prime}\right)$ points for given $M_{\rho}$ 's are also shown in Fig. 9. Obviously, the vector resonance satisfying the existing experimental limits is able to follow the sum rule if its mass stays close or below $1.5 \mathrm{TeV}$.

It is not possible to satisfy all sum rules resulting from the elastic and inelastic $\pi \pi$ scatterings at the same time [31]. In addition, the sum rule motivated by the $\pi \pi \rightarrow h \rho_{L}$ amplitude reads

$a_{V}=a_{\rho}$.

Unfortunately, this is the no-splitting condition not preferred by the data. However, the unitarity-based conclusions reached in [31] rely on the assumption (48). Thus, the results obtained in our analysis call for the investigation of the inelastic unitarity limits when $a_{V} \neq a_{\rho}$. However, this is beyond the scope of this paper and currently work in progress.

\section{The vector resonance mass limits and the upper bounds on $\sigma(p p \rightarrow \rho+X) \times \mathbf{B R}$}

Undeniably, the search for new vector (and other) resonances has its rightful and important place in the ATLAS and CMS Collaboration's activities. While no discovery has been made, the direct exclusion limits constantly improve. Unfortunately, the obtained limits are strongly model and parameter dependent. No wonder that the mass exclusion limits found in the literature cover only some of the interesting cases. To the best of our knowledge, there are no exclusion mass limits applicable to the vector resonance of the model studied in this paper.

One of the crucial factors on which the exclusion mass limit depends is the value of the vector resonance gauge coupling $g^{\prime \prime}$. As we saw in Fig. 9, in our model, the superposition of the unitarity limits over the experimentally preferred region $0.77 \leq a_{V} \leq 1.09$ suggests that considering $g^{\prime \prime}$ below about 10 is not well justified. We cannot rely on the predictions of our Lagrangian with experimentally allowed values of $a_{V}$ when $g^{\prime \prime}=\mathcal{O}(1)$. This is not of great concern to us if the motivation for our model stems from strongly interacting physics. Independently of this restriction, if we narrowed our considerations to the tBESS interaction pattern of the vector resonance to fermions the low-energy limit from the EW precision measurements reads $g^{\prime \prime} \geq 12$ at 95\% CL [35]. This is an additional motivation for considering $g^{\prime \prime}$ values above 10.

We can evaluate how the existing ATLAS and CMS data restrict our model when we compare the predictions of our model with the upper bounds on the resonance production cross section times its branching ratio for various decay channels. The bounds are rather model independent once spin of the resonance under consideration is specified. Of course, one should keep in mind that the calculations involved proceed under the assumption of a narrow-width resonance.

In Table 4, we present the cross section times branching ratios for various decay channels of the considered model at the LHC collision energy of $13 \mathrm{TeV}$. The predictions are given for three different values of the resonance masses, namely $1,1.5$, and $2 \mathrm{TeV}$, and three values of $g^{\prime \prime}$, namely 10 , 15 , and 20 . The $g^{\prime \prime}$ values were chosen to span the region allowed by the combination of the limits considered and obtained in the previous sections. Note that for $g^{\prime \prime}>20$ our theory not only runs into its perturbativity limit, but heavier vector resonances depart from the narrow-width requirement as can be read off of the upper $x$-axes of Fig 9 .

All model predictions quoted in Table 4 correspond to the scenario when the decay of the vector resonance to fermions is negligible. In the tBESS-like fermion sector, this would correspond to turning off the direct coupling of the vector resonance to the third quark generation, ${ }^{9}$ i.e. $b_{L}=b_{R}=0$.

\footnotetext{
9 In the tBESS model [35], there is no direct interaction of the vector resonance with the light fermions. The direct coupling of the vector resonance to the third quark generation is parameterized by $b_{L}$ and $b_{R}$ and by the parameter $p$ which enables the splitting of the direct interactions of the right top and the right bottom quarks. Additional fermion sector parameters $\lambda_{L, R}$ that have been introduced in the tBESS model are, for simplicity, kept at zero values throughout this paper.
} 
Table 4 The production cross section times the branching ratio for different decay channels of the $1,1.5$, and $2 \mathrm{TeV}$ vector resonances of our model considering three different values of $g^{\prime \prime}$. The predictions for the $Z H$ and $W H$ channels are given for $a_{V}=1$ and $a_{\rho}=0$. No direct interactions of the vector resonance with fermions are assumed. The cross sections in the table are calculated for the $13 \mathrm{TeV}$ pp collisions

\begin{tabular}{lllll}
\hline Channel & $g^{\prime \prime}$ & $\sigma\left(\rho^{0}\right)(\mathrm{pb})$ & Channel & $\sigma\left(\rho^{+}\right)+\sigma\left(\rho^{-}\right)(\mathrm{pb})$ \\
\hline$W W$ & 10 & 0.0870 .0160 .005 & $Z W$ & 0.1560 .0300 .009 \\
& 15 & 0.0380 .0070 .002 & & 0.0680 .0130 .004 \\
& 20 & 0.0210 .0040 .001 & & 0.0380 .0070 .002 \\
\hline$Z H$ & 10 & $(712.80 .2) \times 10^{-7}$ & $W H$ & $(2339.70 .9) \times 10^{-7}$ \\
& 15 & $(311.20 .1) \times 10^{-7}$ & & $(1034.30 .4) \times 10^{-7}$ \\
& 20 & $(180.70 .1) \times 10^{-7}$ & & $(572.40 .2) \times 10^{-7}$ \\
\hline$j j(u, d, c, s, b)$ & 10 & $(42716.31 .5) \times 10^{-6}$ & $j j(u, d, c, s)$ & $(57623.12 .2) \times 10^{-6}$ \\
& 15 & $(1887.20 .7) \times 10^{-6}$ & & $(25310.21 .0) \times 10^{-6}$ \\
& 20 & $(1054.00 .4) \times 10^{-6}$ & & $(1425.70 .5) \times 10^{-6}$ \\
\hline$e^{+} e^{-}+\mu^{+} \mu^{-}$ & 10 & $(1074.10 .4) \times 10^{-6}$ & $e v_{e}+\mu v_{\mu}$ & $(1917.80 .7) \times 10^{-6}$ \\
& 15 & $(471.80 .2) \times 10^{-6}$ & & $(843.40 .3) \times 10^{-6}$ \\
& 20 & $(261.00 .1) \times 10^{-6}$ & & $(471.90 .2) \times 10^{-6}$ \\
\hline$t t$ & 10 & $(1054.10 .4) \times 10^{-6}$ & $t b$ & $(28011.31 .1) \times 10^{-6}$ \\
& 15 & $(461.80 .2) \times 10^{-6}$ & & $(1234.90 .5) \times 10^{-6}$ \\
& 20 & $(261.00 .1) \times 10^{-6}$ & & $(692.80 .3) \times 10^{-6}$ \\
\hline
\end{tabular}

Consequently, in this scenario, all fermions can couple to the vector resonance through the mixing-induced interactions only and the decay widths of the neutral/charged vector resonances are dominated by their decays to the EW gauge bosons; $\mathrm{BR}(W W / W Z)>99 \%$.

The experimental upper bounds on the cross section times branching ratio in $W W$ channel read $0.033,0.012$, and $0.005 \mathrm{pb}$ for $M_{\rho}=1,1.5$, and $2 \mathrm{TeV}$, respectively [43]. This excludes the $1 \mathrm{TeV}$ resonance for $g^{\prime \prime} \lesssim 16$ and $1.5 \mathrm{TeV}$ resonance for $g^{\prime \prime} \lesssim 12$. The $2 \mathrm{TeV}$ resonance is unrestricted for $g^{\prime \prime} \geq 10$. Nevertheless, setting $b_{L, R}$ to their maximally lowenergy precision data allowed values $-b_{L, R} \approx 0.1$, as found in $[35]^{10}$ — can lower $\mathrm{BR}(W W)$ of the $1 \mathrm{TeV}$ resonance down to about $70 \%$ for $g^{\prime \prime}=10$, to $30 \%$ for $g^{\prime \prime}=15$, and to $12 \%$ for $g^{\prime \prime}=20$. In the $2 \mathrm{TeV}$ resonance case, $\mathrm{BR}(W W)$ would be lowered to about 97,87 , and $67 \%$, respectively. Thus, we can see that the fermionic interactions of the vector resonance can noticeably decrease the predictions (and, thus, release the experimental restrictions) of the model in this channel. The same effect can be expected to occur in the $Z W$ channel.

Next, let us compare the predictions of our model to the experimental bounds in the $Z W$ channel. The most restrictive bounds in this channel read $0.051 \mathrm{pb}$ [44], $0.022 \mathrm{pb}$ [44], and $0.009 \mathrm{pb}$ [45] for $M_{\rho}=1,1.5$, and $2 \mathrm{TeV}$, respectively. This excludes the $1 \mathrm{TeV}$ resonance for $g^{\prime \prime} \lesssim 17$ and $1.5 \mathrm{TeV}$ resonance for $g^{\prime \prime} \lesssim 12$. Again, the $2 \mathrm{TeV}$ resonance is unre-

\footnotetext{
10 While the preferred value of $p$ found in [35] is about 0.25 , its statistical preference over any other value of $p \in(0,1)$ is marginal. Therefore, we consider $p=1$ in our calculations of the tBESS-like fermion sector contributions, i.e. the same strength of the vector resonance direct couplings to the right top and bottom quarks.
}

stricted for $g^{\prime \prime} \geq 10$. In the case of the tBESS-like fermion sector with $b_{L, R}=0.1$ and $p=1, \mathrm{BR}(W Z)$ gets lowered to about 71,31 , and $12 \%$ for $g^{\prime \prime}=10,15$, and 20 , respectively, when $M_{\rho}=1 \mathrm{TeV}$. When $M_{\rho}=2 \mathrm{TeV}$, the corresponding BR's read 97, 87, and $67 \%$.

The exclusion limits from the $W W / W Z$ channels mentioned in the previous two paragraphs were obtained from $13.2 \mathrm{fb}^{-1}[43,44]$ and $15.5 \mathrm{fb}^{-1}[45]$ of $13 \mathrm{TeV}$ data. For the sake of completeness, we should mention that the combined $W W+W Z$ analysis of $3.2 \mathrm{fb}^{-1}$ of $13 \mathrm{TeV}$ data [46] implies stronger limits $g^{\prime \prime}>13.6$ and $g^{\prime \prime}>10.6$ for $M_{\rho}=1.5$ and $2 \mathrm{TeV}$, respectively.

At tree level, the $Z H$ and $W H$ decays of the vector resonances occur only when $a_{V} \neq a_{\rho}$. In Table 4, we present the predictions of our model for $a_{V}=1$ and $a_{\rho}=0$. They can be used to derive the predictions for preferred values of $a_{V}$ and $a_{\rho}$ that have been found in Sect. 3.3 and quoted in Table 3. Following the formalism introduced in Sect. 2 we find that

$\Gamma_{Z H / W H}\left(a_{V}, a_{\rho}\right)=\left(a_{\rho}-a_{V}\right)^{2} \Gamma_{Z H / W H}(1,0)$

Then, taking into account the negligibility of the contributions of $\Gamma_{Z H}$ and $\Gamma_{W H}$ to the total decay widths of the neutral and charged resonances, the $\left(a_{\rho}-a_{V}\right)^{2}$ scaling applies to the values of the production cross section times the branching ratio for these channels. Thus, when $\left(a_{V}, a_{\rho}\right)=(0.93,-0.08)$, the numbers in the $Z H / W H$ sector of Table 4 are to be multiplied by 1.02 . When $\left(a_{V}, a_{\rho}\right)=$ $(0.93,-1.68),(-0.93,0.52)$, or $(-0.93,2.11)$ the scaling factors are $6.81,2.10$, and 9.24 , respectively. The experimental upper limits on the cross section times the branch- 
ing ratio are $(0.070$ [47], 0.032 [47], 0.013 [48]) $\mathrm{pb}$ for $M_{\rho}=(1.0,1.5,2.0) \mathrm{TeV}$, respectively, in the $Z H$ channel and (0.12 [47], 0.035 [48], 0.013 [48]) pb in the $W H$ channel. The values predicted in these two channels by our model lie some four orders of magnitude below the experimental upper limits. Thus, the upper limits provide no restriction within the considered range of $g^{\prime \prime} \in(10,20)$.

The same conclusions of no restrictions to our model can also be drawn for the remaining decay channels from which the experimental upper bounds for the production cross section times the branching ratio are available. Namely, the upper bounds for the charged $j j$ channel read $0.210 \mathrm{pb}$ and $0.088 \mathrm{pb}$ for $M_{\rho}=1.5$ and $2 \mathrm{TeV}$, respectively, when $15.7 \mathrm{fb}^{-1}$ of $13 \mathrm{TeV}$ data [49] is processed. ${ }^{11}$ The combined neutral+charged $j j$ channel bounds based on $12.9 \mathrm{fb}^{-1}$ of data [50] are 1.20, 0.37, and $0.13 \mathrm{pb}$ for $M_{\rho}=1,1.5$, and $2 \mathrm{TeV}$, respectively. The bounds in the $e^{+} e^{-}+\mu^{+} \mu^{-}$channel based on $13.3 \mathrm{fb}^{-1}$ of data [51] read 1.30, 0.63, and $0.42 \mathrm{fb}$ for $M_{\rho}=1,1.5$, and $2 \mathrm{TeV}$, respectively. The bounds in the $e v_{e}+\mu v_{\mu}$ channel based on $13.3 \mathrm{fb}^{-1}$ of data [52] $\operatorname{read} 4.8,1.8$, and $1.1 \mathrm{fb}$ for $M_{\rho}=1,1.5$, and $2 \mathrm{TeV}$, respectively. The bounds in the $t b$ channel based on $12.9 \mathrm{fb}^{-1}$ of data [53] read 1.8, 0.55, and $0.23 \mathrm{pb}$ for $M_{\rho}=1,1.5$, and $2 \mathrm{TeV}$, respectively. Recall that the values in Table 4 correspond to the scenario with no direct fermion interactions with the vector resonance. In the tBESS-like fermion sector the $t b$ channel production would generally be higher.

Finally, there are also the upper bounds for the $t t$ channel based on $3.2 \mathrm{fb}^{-1}$ of data [54]: $1.20,0.33$, and $0.17 \mathrm{pb}$ for $M_{\rho}=1,1.5$, and $2 \mathrm{TeV}$, respectively. When we compare these bounds with the predictions in Table 4 we can see that for $g^{\prime \prime} \in(10,20)$ they do not restrict our model. It applies even in the case of the tBESS-like fermion sector with $b_{L, R}=$ 0.1 and $p=1$. Then the predictions in the $t t$ channel will rise to about $10^{-2}, 10^{-3}$, and $10^{-4} \mathrm{pb}$ for $M_{\rho}=1,1.5$, and $2 \mathrm{TeV}$, respectively.

\section{Conclusions}

We have studied the experimental and unitarity limits on the parameters of the strong Higgs sector of the phenomenological Lagrangian where beside the composite $125 \mathrm{GeV}$ Higgs boson the $S U(2)_{L+R}$ triplet of composite vector resonances is explicitly present. The ESB sector of our effective Lagrangian has been based on the $S U(2)_{L} \times S U(2)_{R} \rightarrow$ $S U(2)_{L+R}$ non-linear sigma model while the scalar resonance has been introduced as the $S U(2)_{L+R}$ singlet. The vector resonance has been built in employing the hidden local symmetry approach.

$\overline{11}$ The quoted upper bounds for all $j j$ channels include an acceptance factor.
For the interactions of the Higgs boson with the EW gauge fields, the vector triplet, and the top quark the assumed symmetries allow one to introduce three free independent modification factors, $a_{V}, a_{\rho}$, and $c_{t}$. After the transformation from the flavor to mass gauge-boson eigenstate basis, the first two factors combine into modifiers $c_{Z}, c_{W}, c_{\rho^{0}}, c_{\rho^{ \pm}}, c_{Z \rho^{0}}$, and $c_{W \rho^{ \pm}}$of the vertices $h Z Z, h W^{+} W^{-}, h \rho^{0} \rho^{0}, h \rho^{ \pm} \rho^{ \pm}, h Z \rho^{0}$, and $h W^{ \pm} \rho^{\mp}$, respectively. If $r=a_{\rho} / a_{V} \neq 1$, then $c_{Z}$ differs from $c_{W}$. Nevertheless, for quite a large interval of $r$ 's around one, e.g., $|r| \leq 3$, the effect is very small. The corrections to the custodial symmetry protected rho parameter induced by the differing $a_{V}$ and $a_{\rho}$ are negligible and well within the experimental limits. For many phenomenological considerations, the approximations $c_{Z}=c_{W}=a_{V}, c_{\rho^{0}}=c_{\rho^{ \pm}}=a_{\rho}$, and $c_{Z \rho^{0}}=c_{W \rho^{ \pm}}=0$ are satisfactory over quite a large region of $r$ values and for all relevant values of $g^{\prime \prime}$ and $M_{\rho}$.

The limits on the free Higgs coupling factors $a_{V}, a_{\rho}$, and $c_{t}$ have been calculated using constraints on the kappa parameters of the interim framework. The constraints were obtained in the recent ATLAS+CMS Collaborations analysis of various Higgs-related processes based on data from 2011 and 2012. We have used the fitting scenario where no non-SM decays of the Higgs is assumed and where the branching ratio of invisible and/or undetected decay products is zero. In addition, new particles in loops are allowed. Out of seven free parameters in this scenario-loop-level $\kappa_{g}, \kappa_{\gamma}$, and treelevel $\kappa_{W}, \kappa_{Z}, \kappa_{t}, \kappa_{b}, \kappa_{\tau}$-we have used all but the last two to find the restrictions on $a_{V}, a_{\rho}$, and $c_{t} ; \kappa_{b}$ and $\kappa_{\tau}$ have been ignored because their impact on the fitting parameters was negligible.

By fitting the kappas we have established that the simple case of $a_{\rho}=a_{V}$ is strongly disfavored by the data. There are four triplets of the best-fit values of $a_{V}, a_{\rho}$, and $c_{t}$ that can satisfy the fitted data. Namely, $\left(a_{V}, a_{\rho}, c_{t}\right)=$ $(0.93,-0.08,0.85), \quad(0.93,-1.68,0.85),(-0.93,0.52$, $0.85)$, and $(-0.93,2.11,0.85)$. The 1 -sigma deviation (i.e., $\left.\chi_{\min }^{2}+1\right)$ for $a_{V}$ at all four best values is the same: \pm 0.08 . It also applies to $c_{t}$. Its 1 -sigma errors read ${ }_{-0.12}^{+0.11}$ for each best value of $c_{t}$. As far as $a_{\rho}$ is concerned its 1-sigma deviations differ slightly at each of the best-fit values. However, overall they do not exceed \pm 0.17 . The hypothesis backing for all four fits is the same and amounts to $12 \%$. The tied score might be tilted in favor of one of the fits once $\kappa_{Z \gamma}$ gets measured more precisely.

Using scattering amplitudes of the longitudinal EW gauge bosons in the Equivalence theorem approximation we have studied the restrictions of the usability of our phenomenological Lagrangian imposed by the unitarity limits when the data preferred Higgs couplings obtained in this paper are considered. We have found from the $\pi \pi \rightarrow \pi \pi$ scattering that for $M_{\rho}=1 \mathrm{TeV}$ unitarity holds up to at least $\Lambda=3 \mathrm{TeV}$ when $0.77 \leq a_{V} \leq 1.09$ and $12 \leq g^{\prime \prime} \leq 25$. As $M_{\rho}$ grows 
the region where unitarity holds shrinks toward higher $g^{\prime \prime}$ and lower $a_{V}$. Even if $M_{\rho}=2 \mathrm{TeV}$, the considered model is well below the unitarity limit at significant portions of the experimentally allowed region of $a_{V}$. Nevertheless, these conclusions should be complemented by a similar analysis of the $\pi \pi$ scattering with $h$ and $\rho_{L}$ in the final state where $a_{V}$ and $a_{\rho}$ would be independent parameters, thus allowing also for $a_{V} \neq a_{\rho}$ preferred by the data.

Our calculations show that the masses in the range $1 \mathrm{TeV} \leq M_{\rho} \leq 2 \mathrm{TeV}$ are not excluded in parts or even full parameter space of our theory. When the model's predictions face the upper bounds on the production cross section times branching ratio in different decay channels the $1 \mathrm{TeV}$ resonance gets excluded in the $W W$ channel when $g^{\prime \prime} \lesssim 16$ and in the $W Z$ channel when $g^{\prime \prime} \lesssim 17$. Both, the $W W$ and $W Z$ channel measurements exclude the $1.5 \mathrm{TeV}$ resonance when $g^{\prime \prime} \lesssim 14$. These restriction can get weakened once the direct interactions of the vector resonance with the fermion sector are introduced. None of the other reviewed decay channels excludes our model, at least when $10 \leq g^{\prime \prime} \leq 20$.

In the view of the results obtained in this paper we would conclude that even such a simplistic effective description of possible early phenomenology of strong BSM physics as the one studied here is capable to accommodate the existing data.

Acknowledgements We would like to thank F. Riva for useful discussions. The work was supported by the Grants LM2015058 and LG15052 of the Ministry of Education, Youth and Sports of the Czech Republic. J.J. was also supported by the NSP grant of the Slovak Republic. M.G. was supported by the Slovak CERN Fund. We would also like to thank the Slovak Institute for Basic Research for their support.

Open Access This article is distributed under the terms of the Creative Commons Attribution 4.0 International License (http://creativecomm ons.org/licenses/by/4.0/), which permits unrestricted use, distribution, and reproduction in any medium, provided you give appropriate credit to the original author(s) and the source, provide a link to the Creative Commons license, and indicate if changes were made. Funded by SCOAP ${ }^{3}$.

\section{Appendix: Some definitions}

In this appendix, we show definitions of some of the quantities used in Sect. 2 to express the parts of our phenomenological Lagrangian relevant to this paper. All details regarding the Lagrangian structure and how it was built can be found in $[34,35]$.

The field strength tensors of the $S U(2)_{L} \times U(1)_{Y} \times$ $S U(2)_{\text {HLS }}$ gauge fields are defined as

$$
\begin{aligned}
\boldsymbol{W}_{\mu \nu} & =\partial_{\mu} \boldsymbol{W}_{\nu}-\partial_{\nu} \boldsymbol{W}_{\mu}+\left[\boldsymbol{W}_{\mu} \boldsymbol{W}_{\nu}\right], \\
\boldsymbol{B}_{\mu \nu} & =\partial_{\mu} \boldsymbol{B}_{\nu}-\partial_{\nu} \boldsymbol{B}_{\mu},
\end{aligned}
$$

$\boldsymbol{V}_{\mu \nu}=\partial_{\mu} \boldsymbol{V}_{\nu}-\partial_{\nu} \boldsymbol{V}_{\mu}+\left[\boldsymbol{V}_{\mu} \boldsymbol{V}_{\nu}\right]$

where $\boldsymbol{W}_{\mu}=i g W_{\mu}^{a} \tau^{a}, \boldsymbol{B}_{\mu}=i g^{\prime} B_{\mu} \tau^{3}$, and $\boldsymbol{V}_{\mu}=i \frac{g^{\prime \prime}}{2} V_{\mu}^{a} \tau^{a}$ with the gauge couplings $g, g^{\prime}$, and $g^{\prime \prime}$, respectively.

The ESB sector contains six unphysical real scalar fields, would-be Goldstone bosons of the model's spontaneous symmetry breaking. The six real scalar fields $\varphi_{L}^{a}(x), \varphi_{R}^{a}(x), \quad a=1,2,3$, are introduced as parameters of the $S U(2)_{L} \times S U(2)_{R}$ group elements in the expform $\xi\left(\varphi_{L, R}\right)=\exp \left(i \varphi_{L, R} \boldsymbol{\tau} / v\right) \in S U(2)_{L, R}$ where $\boldsymbol{\varphi}=$ $\left(\varphi^{1}, \varphi^{2}, \varphi^{3}\right)$. The quantities $\bar{\omega}_{\mu}^{\|}$and $\bar{\omega}_{\mu}^{\perp}$ are, respectively, the $S U(2)_{L-R}$ and $S U(2)_{L+R}$ projections of the gauged Maurer-Cartan 1-form,

$\bar{\omega}_{\mu}^{\|}=\omega_{\mu}^{\|}+\frac{1}{2}\left(\xi_{L}^{\dagger} \boldsymbol{W}_{\mu} \xi_{L}+\xi_{R}^{\dagger} \boldsymbol{B}_{\mu} \xi_{R}\right)-\boldsymbol{V}_{\mu}$,

$\bar{\omega}_{\mu}^{\perp}=\omega_{\mu}^{\perp}+\frac{1}{2}\left(\xi_{L}^{\dagger} \boldsymbol{W}_{\mu} \xi_{L}-\xi_{R}^{\dagger} \boldsymbol{B}_{\mu} \xi_{R}\right)$,

where $\omega_{\mu}^{\|, \perp}=\left(\xi_{L}^{\dagger} \partial_{\mu} \xi_{L} \pm \xi_{R}^{\dagger} \partial_{\mu} \xi_{R}\right) / 2$.

\section{References}

1. G. Aad et al., ATLAS Collaboration. Phys. Lett. B 716, 1 (2012)

2. S. Chatrchyan et al., CMS Collaboration. Phys. Lett. B 716, 30 (2012)

3. R. Barbieri, B. Bellazini, V.S. Rychkov, A. Varagnolo, Phys. Rev. D 76, 115008 (2007)

4. C. Csaki, A. Falkowski, A. Weiler, JHEP 0809, 008 (2008)

5. R. Contino. arXiv:1005.4269 [hep-ph]

6. R. Contino, D. Marzocca, D. Pappadopulo, R. Rattazzi, JHEP 1110, $081(2011)$

7. A. Pomarol, F. Riva, JHEP 1208, 135 (2012)

8. D. Pappadopulo, A. Thamm, R. Torre, JHEP 1307, 058 (2013)

9. M. Montull, F. Riva, E. Salvioni, R. Torre, Phys. Rev. D 88, 095006 (2013)

10. G. Panico, A. Wulzer, Lect. Notes Phys. 913, 1 (2016). arXiv: 1506.01961

11. W.A. Bardeen, C.T. Hill, M. Lindner, Phys. Rev. D 41, 1647 (1990)

12. G.F. Giudice, C. Grojean, A. Pomarol, R. Rattazzi, JHEP 0706, 045 (2007)

13. R. Foadi, M.T. Frandsen, T.A. Ryttov, F. Sannino, Phys. Rev. D 76, 055005 (2007)

14. T.A. Ryttov, F. Sannino, Phys. Rev. D 78, 115010 (2008)

15. F. Sannino, Acta Phys. Polon. B 40, 3533 (2009)

16. A.R. Zerwekh, Mod. Phys. Lett. A 25, 423 (2010)

17. A.E. Cárcamo Hernández, R. Torre, Nucl. Phys. B 841, 188 (2010)

18. G. Burdman, C.E.F. Haluch, JHEP 1112, 038 (2011)

19. T. Hapola, F. Sannino, Mod. Phys. Lett. A 26, 2313 (2011)

20. A.E. Cárcamo Hernández, C.O. Dib, N. Neill, A.R. Zerwekh, JHEP 1202, 132 (2012)

21. R. Foadi, M.T. Frandsen, F. Sannino, Phys. Rev. D 87, 095001 (2013)

22. R. Contino, M. Ghezzi, C. Grojean, M. Muhlleitner, M. Spira, JHEP 1307, 035 (2013)

23. O. Castillo-Felisola, C. Corral, M. González, G. Moreno, N.A. Neill, F. Rojas, J. Zamora, A.R. Zerwekh, Eur. Phys. J. C 73, 2669 (2013)

24. A.E. Cárcamo Hernández, C.O. Dib, A. R. Zerwekh, Eur. Phys. J. C 74, 2822 (2014) 
25. A.E. Cárcamo Hernández, C.O. Dib, A. R. Zerwekh, Nucl. Part. Phys. Proc. 35, 267-269 (2015)

26. D. Pappadopulo, A. Thamm, R. Torre, A. Wulzer, JHEP 1409, 060 (2014)

27. A. Belyaev, M.S. Brown, R. Foadi, M.T. Frandsen, Phys. Rev. D 90, 035012 (2014)

28. B.W. Lee, C. Quigg, H.B. Thacker, Phys. Rev. D 16, 1519 (1977)

29. B.W. Lee, C. Quigg, H.B. Thacker, Phys. Rev. Lett. 38, 883 (1977)

30. M.S. Chanowitz, M.K. Gaillard, Nucl. Phys. B 261, 379 (1985)

31. B. Bellazzini et al., JHEP 11, 003 (2012)

32. M. Bando, T. Kugo, K. Yamawaki, Phys. Rep. 164, 217 (1988)

33. R.S. Chivukula et al., Phys. Rev. D 74, 075011 (2006)

34. M. Gintner, J. Juráň, I. Melo, Phys. Rev. D 84, 035013 (2011)

35. M. Gintner, J. Juráň, Eur. Phys. J. C 73, 2577 (2013)

36. R. Casalbuoni, S. De Curtis, D. Dominici, R. Gatto, Phys. Lett. 155B, 95 (1985)

37. R. Casalbuoni, S. De Curtis, D. Dominici, R. Gatto, Nucl. Phys. B 282, 235 (1987)

38. R. Casalbuoni, P. Chiappetta, S. De Curtis, F. Feruglio, R. Gatto, B. Mele, J. Terron, Phys. Lett. B 249, 130 (1990)
39. The ATLAS and CMS Collaborations. arXiv:1606.02266

40. The LHC Higgs Cross Section Working Group. arXiv:1307.1347

41. The LHC Higgs Cross Section Working Group. arXiv:1101.0593

42. ATLAS Collaboration, Eur. Phys. J. C 76, 6 (2016)

43. ATLAS Collaboration, ATLAS-CONF-2016-062

44. ATLAS Collaboration, ATLAS-CONF-2016-082

45. ATLAS Collaboration, ATLAS-CONF-2016-055

46. ATLAS Collaboration. arXiv: 1606.04833

47. ATLAS Collaboration. arXiv:1607.05621

48. ATLAS Collaboration, ATLAS-CONF-2016-083

49. ATLAS Collaboration, ATLAS-CONF-2016-069

50. CMS Collaboration, CMS-PAS-EXO-16-032

51. ATLAS Collaboration, ATLAS-CONF-2016-045

52. ATLAS Collaboration, ATLAS-CONF-2016-061

53. CMS Collaboration, CMS-PAS-B2G-16-017

54. ATLAS Collaboration, ATLAS-CONF-2016-014 